\title{
Microwave Photon-Mediated Interactions between Semiconductor Qubits
}

\author{
D. J. van Woerkom, ${ }^{*}$ P. Scarlino, J. H. Ungerer, C. Müller, J. V. Koski, A. J. Landig, C. Reichl, \\ W. Wegscheider, T. Ihn, K. Ensslin, and A. Wallraff ${ }^{\dagger}$ \\ Department of Physics, ETH Zurich, CH-8093 Zurich, Switzerland
}

(Received 21 June 2018; revised manuscript received 20 September 2018; published 31 October 2018)

\begin{abstract}
The realization of a coherent interface between distant charge or spin qubits in semiconductor quantum dots is an open challenge for quantum information processing. Here, we demonstrate both resonant (real) and nonresonant (virtual) photon-mediated coherent interactions between double quantum-dot charge qubits separated by several tens of micrometers. We present clear spectroscopic evidence of the resonant collective enhancement of the coupling of two qubits and the resonator. With both qubits in resonance with each other but detuned from the resonator, we observe exchange coupling between the qubits mediated by virtual photons. In both instances, pronounced bright and dark states governed by the symmetry of the qubit-field interaction are found. Our observations are in excellent quantitative agreement with masterequation simulations. The extracted two-qubit coupling strengths significantly exceed the linewidths of the combined resonator-qubit system, which indicates that this approach is viable for creating photon-mediated two-qubit gates in quantum-dot-based systems.
\end{abstract}

DOI: 10.1103/PhysRevX.8.041018

Semiconductor nanostructure-based systems are one of the promising contenders for quantum information processing, since they offer flexibility in tuning, long coherence times, and well-established fabrication techniques [1,2]. However, scaling to larger numbers of qubits remains a challenge, since many coupling mechanisms for realizing two-qubit gates are short range, i.e., limited to nearest neighbors. For scaling to larger systems and eventually to a full-scale quantum computer, a combination of short- and longer-range interactions seems promising [3].

So far, short-range (approximately $100 \mathrm{~nm}$ ) qubit-qubit interaction has been realized via capacitive or exchange coupling between charge [4-6] and spin qubits [7-10], which is expanded by making use of interactions mediated by additional qubits (approximately $400 \mathrm{~nm}$ ) [11] or electronic cavities (approximately $1.7 \mu \mathrm{m}$ ) [12]. In addition, distant coupling by transferring electrons using surface acoustic waves [13] or by modulating charge gates $[14,15]$ are being investigated. However, it is predicted

\footnotetext{
${ }^{\dagger}$ Corresponding author. andreas.wallraff@phys.ethz.ch

Corresponding author. andreas.wallraff@phys.ethz.ch

Published by the American Physical Society under the terms of the Creative Commons Attribution 4.0 International license. Further distribution of this work must maintain attribution to the author(s) and the published article's title, journal citation, and DOI.
}

Subject Areas: Quantum Physics,

Quantum Information,

Semiconductor Physics

that the range of interaction between semiconductor qubits can be increased significantly using microwave photons $[3,16,17]$. A key ingredient, the strong coupling of individual charges $[18,19]$ or spins $[20,21]$ to individual microwave photons, has recently been realized in semiconductor implementations of circuit quantum electrodynamics (QED) [22].

Here, we present experiments in which the coherent photon-mediated coupling between two spatially separated semiconductor qubits is realized in both the resonant and the dispersive regime using high-impedance SQUID array resonators. The high Josephson inductance and low capacitance to ground of the SQUID array increases the resonator impedance and, thus, the strength of the vacuum fluctuations of the electric field. The use of a high-impedance resonator enhances the coupling strength of the individual qubits to the resonator [19] and consequently the qubit-qubit coupling, which allows us to overcome the limitations of prior experiments $[20,23,24]$. This key step holds the strong promise that two-qubit gates based on photon-mediated interactions, which are a cornerstone in quantum information processing with superconducting circuits [25], are implementable with semiconductor qubits based on a variety of material systems.

In this work, we investigate two semiconductor doublequantum-dot (DQD) charge qubits strongly coupled via one of its plunger gates to a single high-impedance resonator [Figs. 1(a) and 1(b)] composed of 35 SQUIDS with an estimated impedance of approximately $1 \mathrm{k} \Omega$ [19]. At the end of the flux tunable resonator, the DQDs are defined using depletion gate technology on a mesa of a GaAs/AlGaAs 
heterostructure. They are separated by a distance of $42 \mu \mathrm{m}$, much larger than in conventional multi-quantum-dot devices. In this architecture, the separation between the quantum dots could straightforwardly be extended to several hundred micrometers at the cost of increasing the stray capacitance and reducing the coupling strength. In superconducting circuits, qubits are routinely coupled over millimeter to centimeter distances [26]. Even larger distances have recently been achieved by exchanging shaped photons through meter-long transmission lines [27-29]. Thus, we also expect the separation between semiconductor qubits to be extendable to these scales. For our experiment, the chosen distance is a trade-off in realizing a much larger separation than was achieved with exchange or capacitive coupling and still realizing the strong coupling limit.

Both DQD charge qubits are coupled to the antinode of the electric field at the open end of the resonator [Figs. 1(c)1(e)]. The resonator is designed for readout and additionally acts as a coupler between the spatially separated DQDs. The design and fabrication are similar to the one described in Refs. $[19,30]$ and are discussed in detail in Appendix A.

We characterize the properties of the device by measuring the amplitude $\left|S_{11}\right|$ and phase $\varphi$ of a microwave tone reflected off the resonator at the drive line indicated in
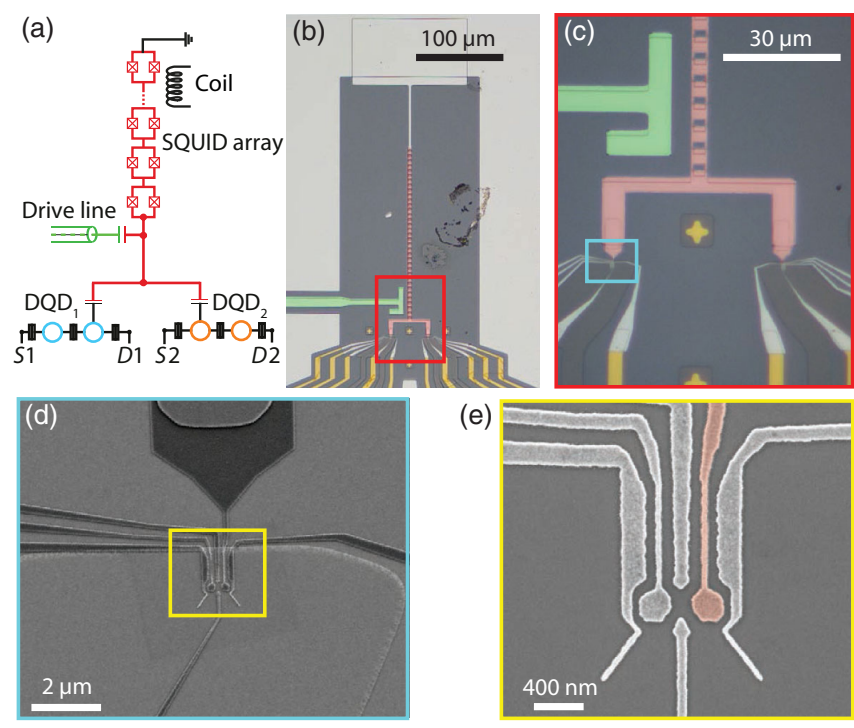

(e)

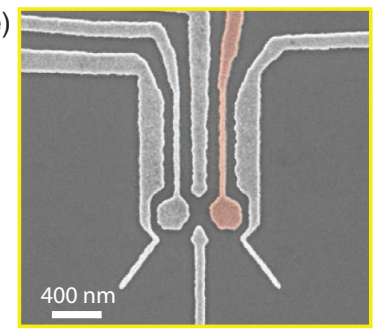

FIG. 1. Simplified circuit diagram and micrograph of the device. (a) Schematic of the device and control line: SQUID array resonator (red), drive line (green), two DQDs (cyan and orange), and an external coil (black). The color code is used throughout this paper. (b) False-color optical micrograph of the measured device. (c) Detail of (b) showing the resonator and its drive line coupled to both DQDs. (d) Scanning electron micrograph (SEM) of the resonator connected to $\mathrm{DQD}_{1}$ on the left. $\mathrm{DQD}_{2}$ is defined as a mirrored copy of $\mathrm{DQD}_{1}$, separated from it by $42 \mu \mathrm{m}$. (e) SEM micrograph of the gate structure used for defining the DQDs in the GaAs/AlGaAs heterostructures. (d) and (e) are images of identically designed devices not used in the experiments. green in Fig. 1. The same line is also used to apply microwave spectroscopy tones to the individual qubits (see Appendix B for a complete description of the measurement setup).

With the qubit transition frequencies far detuned from the resonator [19] operated at $\omega_{r} / 2 \pi=5.171 \mathrm{GHz}$, we spectroscopically determine the resonator internal loss rate $\kappa_{\text {int }} / 2 \pi=17 \mathrm{MHz}$ and its external coupling rate $\kappa_{\text {ext }} / 2 \pi=6 \mathrm{MHz}$, governed by the coupling to the drive line. This result puts the resonator into the weakly undercoupled regime $\left(\kappa_{\text {int }}>\kappa_{\text {ext }}\right)$, keeping the total resonator linewidth small. The internal losses are dominated by the residual coupling to the gate leads [31], which is not mitigated by in-line filters in our device and by the piezoelectricity of the GaAs substrate. We configure the two DQDs $(k=1,2)$ as two-level systems described in good approximation by the Hamiltonian $H_{k}=$ $-\delta_{k} \sigma_{z} / 2+t_{k} \sigma_{x}$ with Pauli matrices $\sigma_{x, y, z}$. The transition frequency of each quantum-dot qubit $\omega_{\mathrm{DQD}-k}=\sqrt{4 t_{k}^{2}+\delta_{k}^{2}}$ is a hyperbolic function of detuning $\delta_{k}$ between the charge states of the individual dots and tunnel rate $2 t_{k}$ between them.

We tune $2 t_{1} \sim \omega_{r}$ such that $\mathrm{DQD}_{1}$ is in resonance with the high-impedance resonator at $\delta_{1}=0$. We first measure the reflection spectrum $\left|S_{11}\right|$ of the resonator tuning $\delta_{1}$ of the left DQD, labeled $\mathrm{DQD}_{1}$ in Fig. 1(a), from negative to positive values with the second DQD far detuned, $\delta_{2} \gg \omega_{r}$. We observe a well-resolved vacuum Rabi mode splitting [19] with a coupling rate of $g_{1} / 2 \pi=53 \mathrm{MHz}$. The photon state of the resonator and the charge state of the DQD hybridize in a resonant twobody (anti)symmetric state, $| \pm\rangle_{r 2}=(|e, 0\rangle \pm|g, 1\rangle) / \sqrt{2}$ [32] as illustrated in Fig. 2(f) with the charge qubit ground $|g\rangle$ and excited state $|e\rangle$ and the cavity photon number states $|0\rangle$ and $|1\rangle$. We independently determined the linewidth $\Gamma_{2,1} / 2 \pi=4.8 \pm 0.6 \mathrm{MHz}$ of $\mathrm{DQD}_{1}$ at this frequency using qubit spectroscopy in the dispersive regime, making use of the tunable resonator [19]. Equivalent measurements are performed for $\mathrm{DQD}_{2}$, adjusting its bias configuration to reach a coupling strength $g_{2} / 2 \pi=56 \mathrm{MHz}$, similar to $\mathrm{DQD}_{1}$, and finding $\Gamma_{2,2} / 2 \pi=5.6 \pm 0.5 \mathrm{MHz}$; see Appendix D. These results shows that each DQD is individually strongly coupled to the resonator: $g_{k}>\left(\kappa_{\text {int }}+\kappa_{\text {ext }}\right) / 2+\Gamma_{2, k}$.

We model the coupled system using a master-equation simulation with the Tavis-Cummings Hamiltonian

$$
H=\omega_{r} a^{\dagger} a+\sum_{k} H_{k}+\sum_{k} g_{k} \sigma_{z}\left(a^{\dagger}+a\right),
$$

with $\hbar=1$, bosonic annihilation (creation) operators $a$ $\left(a^{\dagger}\right)$, and the coupling rate $g_{k}$ between the resonator and $\mathrm{DQD}_{k}$ (Appendix C). The observed resonance frequencies and linewidths are in excellent agreement with the simulation (dashed lines in Fig. 2), which allow us to extract the system parameters with high accuracy (Appendix H). 

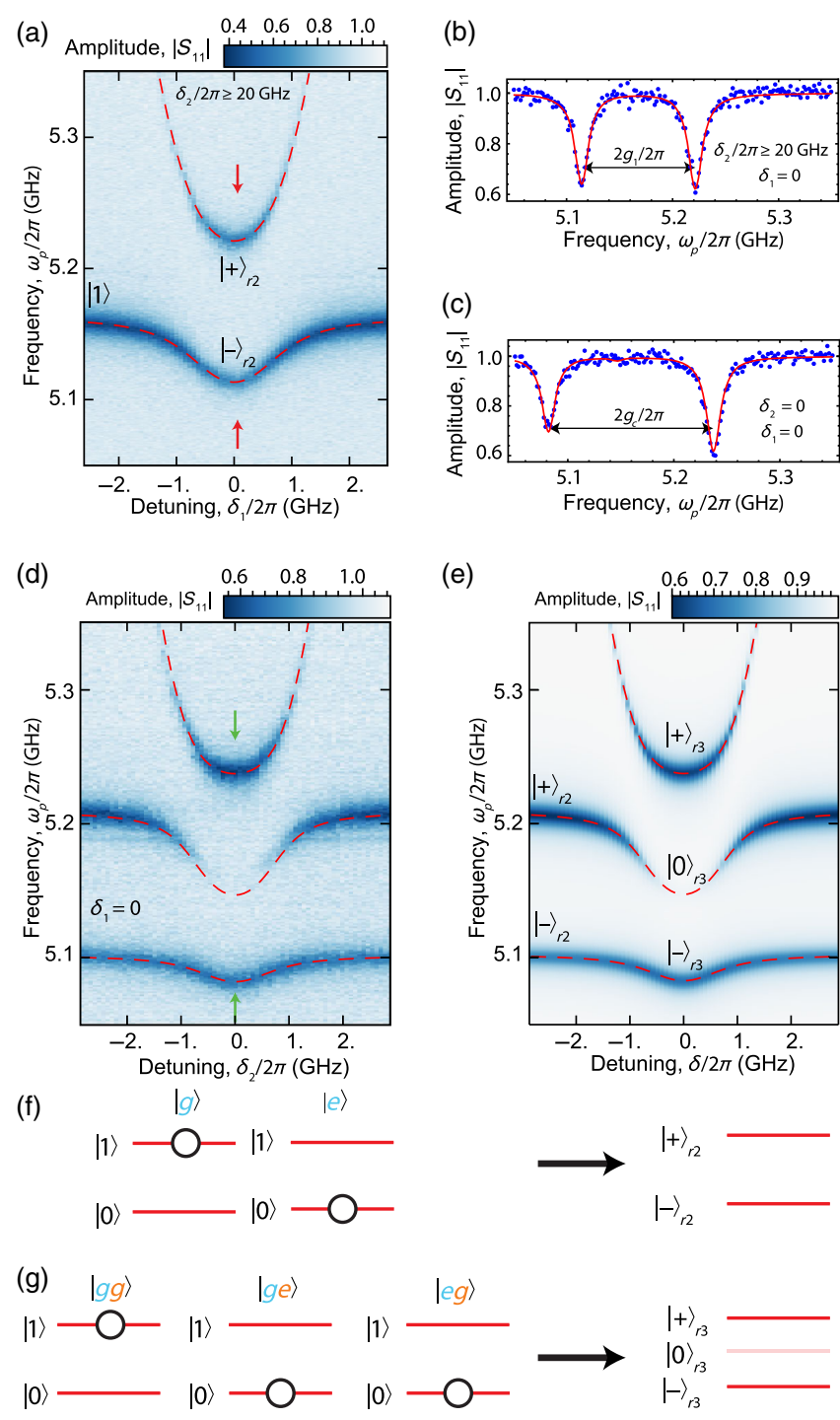

FIG. 2. Individual and two-qubit vacuum Rabi mode splitting. (a) Measured reflection coefficient $\left|S_{11}\right|$ vs drive frequency $\omega_{p}$ and charge detuning $\delta_{1}$ for $\mathrm{DQD}_{1}\left(2 t_{1} / 2 \pi=5.166 \mathrm{GHz}\right)$ tuned into resonance with the SQUID array resonator $\left(\omega_{r} / 2 \pi=\right.$ $5.171 \mathrm{GHz}$ ). The red dashed lines are extracted from fits to a master equation model; see the text for details. (b) $\left|S_{11}\right|\left(\omega_{p}\right)$ at $\delta_{1}=0$ [red arrows in (a)]. (c) $\left|S_{11}\right|\left(\omega_{p}\right)$ at $\delta_{1} \sim \delta_{2} \sim 0$ [green arrows in (d)]. (d) $\left|S_{11}\right|$ vs drive frequency $\omega_{p}$ and charge detuning $\delta_{2}$ for $\mathrm{DQD}_{1}$ biased at $2 t_{1} / 2 \pi=5.166 \mathrm{GHz}$ and $\delta_{1} \approx 0$ and $\mathrm{DQD}_{2}$ at $2 t_{2} / 2 \pi=5.156 \mathrm{GHz}$ realizing $\omega_{r}=$ $\omega_{\mathrm{DQD} 1}=\omega_{\mathrm{DQD} 2}$ at $\delta_{1,2} \approx 0$. (e) Master-equation simulation of $\left|S_{11}\right|\left(\delta_{2}, \omega_{p}\right)$ fitting to the data in (d), details in Appendix C. Schematic energy-level diagram of (f) one and ( $g$ ) two DQDs interacting with the resonator. Basis states are shown on the left, coupled states on the right; see the text and Appendix C for state labeling.

We now explore the case of all three transitions tuned into mutual resonance $\left(\omega_{r}=\omega_{\mathrm{DQD1}}=\omega_{\mathrm{DQD} 2}\right)$ by measuring the reflection spectrum $\left|S_{11}\right|$ of $\mathrm{DQD}_{1}$ resonantly coupled to the resonator $\left(2 t_{1}=\omega_{r}, \delta_{1}=0\right)$ and tuning
$\mathrm{DQD}_{2}$ into resonance using its charge detuning parameter $\delta_{2}$. We observe the transition of a single-qubit vacuum Rabi mode-splitting spectrum at large detunings $\delta_{2}$ to a wellresolved two-qubit vacuum Rabi mode-splitting spectrum [Fig. 2(d)] at $\delta_{2}=0$, with the collectively enhanced twoqubit coupling rate $g_{c} / 2 \pi=\sqrt{g_{1}^{2}+g_{2}^{2}} / 2 \pi=77 \mathrm{MHz}$ [Fig. 2(c)]. This result is a clear signature of the coherent photon-mediated coupling between two spatially separated DQDs in the resonant regime.

On resonance, the three systems $(r 3)$ form a triplet of two bright states $| \pm\rangle_{r 3}=\left(g_{2}|g, e, 0\rangle+g_{1}|e, g, 0\rangle \pm\right.$ $\left.g_{c}|g, g, 1\rangle\right) / \sqrt{2} g_{c}$ and one dark state $|0\rangle_{r 3}=\left(g_{1}|g, e, 0\rangle-\right.$ $\left.g_{2}|e, g, 0\rangle\right) / g_{c} \quad$ at frequencies $\omega_{| \pm\rangle_{r 3}}=\omega_{r} \pm g_{c}$ and $\omega_{|0\rangle_{r 3}}=\omega_{r}$; see the schematic in Fig. 2(g) and Appendix C. This feature occurs because the drive field acts symmetrically on both qubits, exciting only the symmetric qubit superposition of the bright states but not the antisymmetric superposition of the dark state [26,33]. The data in Fig. 2(d) show excellent quantitative agreement with the master-equation model; see the dashed red lines indicating the transition frequencies between the ground and the joint excited states allowing us to extract all relevant system parameters [Figs. 2(d) and 2(e)].

Alternatively, coherent coupling between spatially separated DQDs can be mediated by virtual photons. The DQD qubit transitions are tuned in resonance but detuned $\left(\Delta_{r}=\omega_{r}-\omega_{\mathrm{DQD}}\right)$ from the resonator; one DQD excitation can be transferred to the other DQD by virtually populating the resonator with a photon. In this case, the effective coupling strength is reduced, but the coupling mechanism is insensitive to photon loss from the resonator.

To observe the dispersive coupling, we tune the resonator to $\omega_{r} / 2 \pi=5.454 \mathrm{GHz}$, resulting in a detuning $\Delta_{r} / 2 \pi \approx$ $300 \mathrm{MHz}$ when both qubits are at $\delta_{k}=0$. The virtual photon-mediated exchange coupling is observed by the formation of a dark and bright state split in energy [Fig. 3(a)] when the two DQDs are (approximately) in resonance $\omega_{\mathrm{DQD} 1} \sim \omega_{\mathrm{DQD} 2}$. This observation also allows us to identify the resonances as transitions to the dispersively coupled two-qubit entangled states $| \pm\rangle_{d 2}=\left(g_{1}|g, e\rangle \mp\right.$ $\left.g_{2}|e, g\rangle\right) / g_{c}$ [26] (Fig. 3). Because of the near-equal coupling rates $g_{1} \sim g_{2}$, the dark state is fully developed when the DQDs are resonant, $\omega_{\mathrm{DQD} 1}=\omega_{\mathrm{DQD} 2}$ (see Appendix C). Then, only the bright state is directly observable in qubit spectroscopy; see the line traces in Appendix G. To observe the splitting directly, we can instead bias the DQDs to achieve $g_{1} \neq g_{2}$, which through the asymmetry in parameters makes the otherwise dark state observable.

We therefore configure both DQDs at a new charge bias point at which the coupling rates to the resonator are $g_{1} / 2 \pi=34 \mathrm{MHz}$ and $g_{2} / 2 \pi=69 \mathrm{MHz}$ (see Appendix E). At $\delta_{1,2} \sim 0$, the qubit linewidths $\Gamma_{2,1} / 2 \pi=(4.6 \pm 0.6) \mathrm{MHz}$ and $\Gamma_{2,2} / 2 \pi=(6.3 \pm 1.1) \mathrm{MHz}$ are determined from 

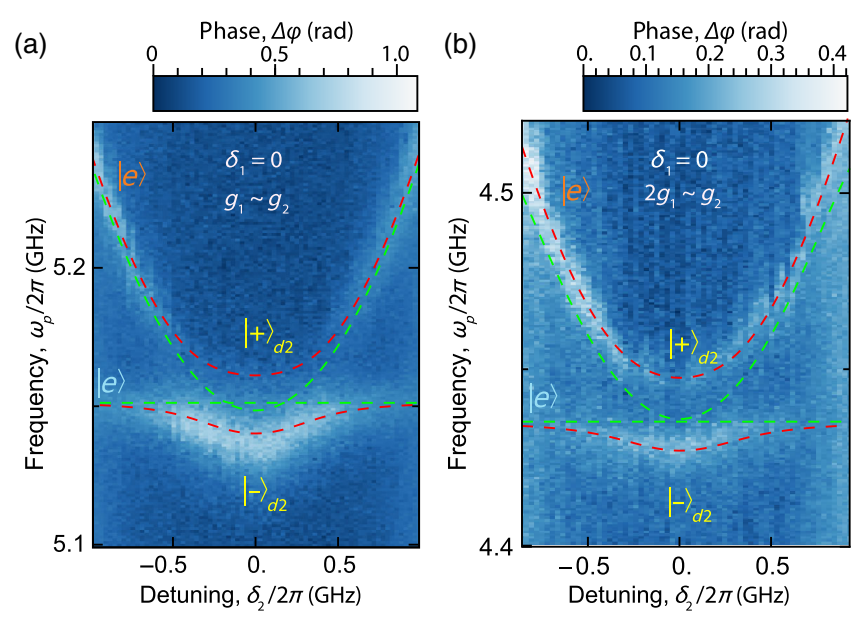

(c)

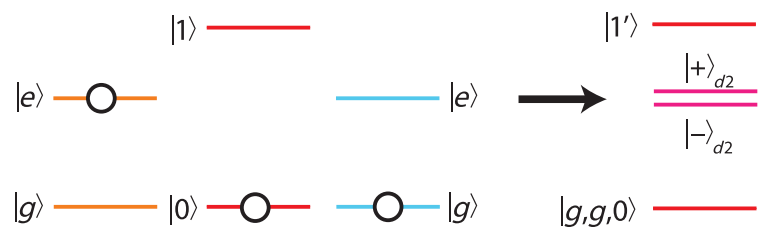

FIG. 3. Qubit spectroscopy of the virtual photon-mediated qubitqubit exchange interaction. (a) Resonator phase shift $\Delta \varphi$ for DQDs with equal coupling $\left(g_{1} \sim g_{2}\right)$ at fixed $2 t_{1} / 2 \pi=5.156 \mathrm{GHz}$, $\delta_{1}=0$, and $2 t_{2} / 2 \pi=5.148 \mathrm{GHz}$ vs detuning parameter $\delta_{2}$. Line traces and additional data are in Appendix G. (b) Qubit spectroscopy for DQDs with different coupling $\left(2 g_{1} \sim g_{2}\right)$ and $\omega_{\mathrm{DQD} 1} / 2 \pi\left(\delta_{1}=0\right)=4.436 \mathrm{GHz}$ measuring the phase shift $\Delta \varphi$ of the resonator vs $\delta_{2}$ for $2 t_{2} / 2 \pi=4.443 \mathrm{GHz}$. (c) Schematic energylevel diagram of two DQDs in resonance and a detuned resonator. Basis states are shown on the left and hybridized states on the right with labels discussed in the text and in Appendix C.

spectroscopy measurements with the other qubit largely detuned. For these measurements, the resonator is tuned to $\omega_{r} / 2 \pi=4.717 \mathrm{GHz}\left(\kappa_{\text {int }} / 2 \pi=8 \mathrm{MHz}, \kappa_{\text {ext }} / 2 \pi=4 \mathrm{MHz}\right)$.

Next, we perform qubit spectroscopy with $2 t_{1} / 2 \pi \sim$ $2 t_{2} / 2 \pi \sim 4.44 \mathrm{GHz}$, corresponding to a detuning $\Delta_{r} / 2 \pi \approx$ $280 \mathrm{MHz}$ of each DQD from the resonator at $\delta_{1,2}=0$, putting the system in the dispersive regime [22]. A virtual photon-mediated exchange coupling $2 \mathrm{~J} / 2 \pi=24.8 \mathrm{MHz}$ is observed spectroscopically when varying the detuning $\delta_{2}$ and keeping the bias parameters of $\mathrm{DQD}_{1}$ fixed [Fig. 3(b)]. The system parameters which are used to display the noninteracting transition frequencies (green dashed lines) in Figs. 3(a) and 3(b) are extracted from a master-equation simulation (red dashed lines).

We note that the spectroscopic lines of $\mathrm{DQD}_{1}$ at large detuning $\left|\delta_{2} / 2 \pi\right| \gtrsim 0.5 \mathrm{GHz}$ are less pronounced, due to its weaker coupling to the resonator and because $\mathrm{DQD}_{2}$ is dispersively shifting the resonator, rendering the readout less sensitive for $\mathrm{DQD}_{1}$.

Finally, we determine the scaling of the exchange coupling $J$ with detuning $\Delta_{r}$ from the flux-tuned resonator for the same fixed qubit parameters at $\delta_{1,2}=0$ [Fig. 4(b)]. We use the configuration in Fig. 3(b), since both symmetric and
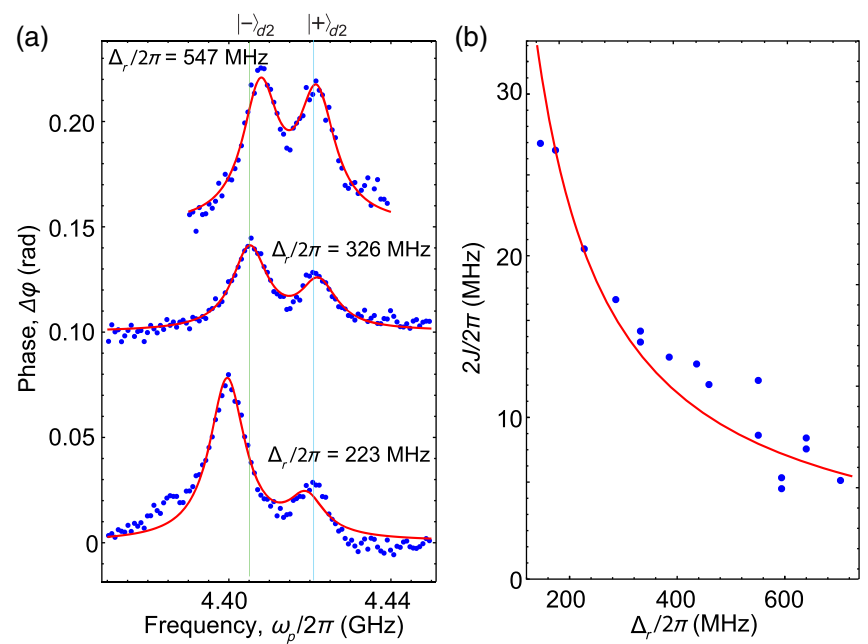

FIG. 4. Coherent qubit-qubit exchange splitting $2 J$ vs resonator detuning $\Delta_{r}(\Phi)$. (a) Resonator phase shift (data points offset for clarity) measurement performed to extract qubit-qubit interaction $2 J$ on resonance for the indicated detunings $\Delta_{r}(\Phi)$. Solid lines are fits to master-equation simulations; see Appendixes $\mathrm{C}$ and $\mathrm{H}$ for details and parameters. (b) $2 J$ extracted from (b) and similar data vs $\Delta_{r}$. The solid line is a fit to $1 / \Delta_{r}$.

antisymmetric resonances are observable for $\omega_{\mathrm{DQD} 1}=$ $\omega_{\mathrm{DQD} 2}$ which allow us to extract $2 J$ by fitting to results of the master-equation simulation [Fig. 4(a)]. At small resonator detunings, we find the largest coherent qubit-qubit exchange rates of $2 \mathrm{~J} / 2 \pi=27 \mathrm{MHz}$ [Fig. 4(b)], clearly exceeding the combined qubit linewidths $\left(\Gamma_{2,1}+\Gamma_{2,2}\right) / 2 \pi=11 \mathrm{MHz}$. For $\Delta_{r} / 2 \pi>560 \mathrm{MHz} \sim 8 g_{2} / 2 \pi$, the $2 J$ is smaller than the qubit linewidths.

We note that the transition of the dark state $|+\rangle_{d 2}$ remains at a fixed frequency while the bright state $|-\rangle_{d 2}$ shifts as a function of $\Delta_{r}$ in agreement with our master-equation model [Fig. 4(a)]; see Appendix C. When plotting $2 J$ vs the resonator detuning $\Delta_{r}(\Phi)$, we find approximately the expected scaling with $1 / \Delta_{r}$ [Fig. 4(b)]. Also, the overall coupling strength $g_{1} g_{2} / 4 \pi^{2}=2.1 \times 10^{3} \mathrm{MHz}^{2}$ is consistent with the one calculated $\left(2.4 \times 10^{3} \mathrm{MHz}^{2}\right)$ from the individually measured qubit-resonator coupling rates $g_{1,2}$ and the detuning $\Delta_{r}$.

We emphasize that the device investigated here features a frequency-tunable resonator $\left(\omega_{r}\right)$, charge qubits with a tunable transition frequency $(\delta)$, and sweet-spot $(2 t)$ and dipole coupling strength $(g)$ enabling a comprehensive study of coherent photon-mediated coupling phenomena, the concepts of which are transferable to other semiconductor material systems. We also point out that photon-mediated coupling enables two-qubit gates between charge or spin qubits across micrometer, millimeter, or even longer distances, which is essential for scaling quantum information processing with semiconductor qubits $[3,16]$. For spin qubits, high-impedance resonators which are magnetic-field compatible (see, e.g., Ref. [34]) would need to be employed in combination with micromagnets 
(see, e.g., Refs. [20,21]). In superconducting circuits, the observation of long-range qubit-qubit coupling [26,33] led to the development of both resonant and dispersive photonmediated two-qubit gates $[35,36]$ and enabled the scaling of circuits to the level of several tens of qubits [25].

The data presented in this paper and corresponding supplemental material will be available online at ETH Zurich repository for research data [37].

We acknowledge contributions by C. K. Andersen, S. Gasparinetti, M. Collodo, J. Heinsoo, S. Storz, M. Frey, A. Stockklauser, and M. Gabureac. We thank Ataç İmamoğlu and Gianni Blatter for valuable feedback on the manuscript. This work was supported by the Swiss National Science Foundation (SNF) through the National Center of Competence in Research (NCCR) Quantum Science and Technology (QSIT), the project Elements for Quantum Information Processing with Semiconductor/ Superconductor Hybrids (EQUIPS), and by ETH Zurich.

P. S., J. H. U., and J. V. K. designed the device with input from A. W. D. J. v. W., P. S., and J. V. K. fabricated the device. Electrical and microwave measurements and data analysis were performed by D. J. v. W., P. S., and J. H. U. The theoretical model and fits were done by C. M. A. J. L., T. I., and K. E. had valuable input to the experiments. C. R. and $\mathrm{W}$. W. grew the GaAs heterostructure. The manuscript was written by D. J. v. W., C. M., and A. W. with comments from all authors. A. W. supervised the project.

\section{APPENDIX A: DEVICE AND FABRICATION}

The substrate is a commercially available GaAs wafer with a 500-nm GaAs layer grown by molecular beam epitaxy. Subsequently, a 40-nm layer of $\mathrm{Al}_{x} \mathrm{Ga}_{1-x} \mathrm{As}$ is grown as a spacer to a $\delta$-donor layer of silicon dopants followed by $45 \mathrm{~nm}$ of $\mathrm{Al}_{x} \mathrm{Ga}_{1-x}$ As and capped by a $5-\mathrm{nm}$ GaAs layer. $90 \mathrm{~nm}$ below the surface, at the interface of $\mathrm{GaAs} / \mathrm{Al}_{x} \mathrm{Ga}_{1-x} \mathrm{As}$, a two-dimensional electron gas (2DEG) is formed by bending the conduction band below the Fermi energy.

In the first photolithography step, a GaAs mesa hosting the DQDs is formed by wet etching with a piranha solution. The source and drain up to the DQDs are also formed as part of the mesa. We emphasize that all 2DEG is removed below the SQUID array resonator to maintain its quality factor. The fabrication residue visible in the resonator area, in Fig. 5, could not be removed. We believe that this residue does not lead to any reduction in device performance, as the internal loss rate of the resonator is similar in previous devices $[19,30]$. In the subsequent photolithography lift-off step, the Ohmic contact of the DQD source and drain are deposited by electron beam evaporation of a $\mathrm{Ge} / \mathrm{Au} / \mathrm{Ni}$ layer, which are annealed at $470^{\circ} \mathrm{C}$ for $5 \mathrm{~min}$ to diffuse into the 2DEG layer.

The electrostatic gates are created in two lithography steps. First, the coarse gates and pads [yellow (gold)

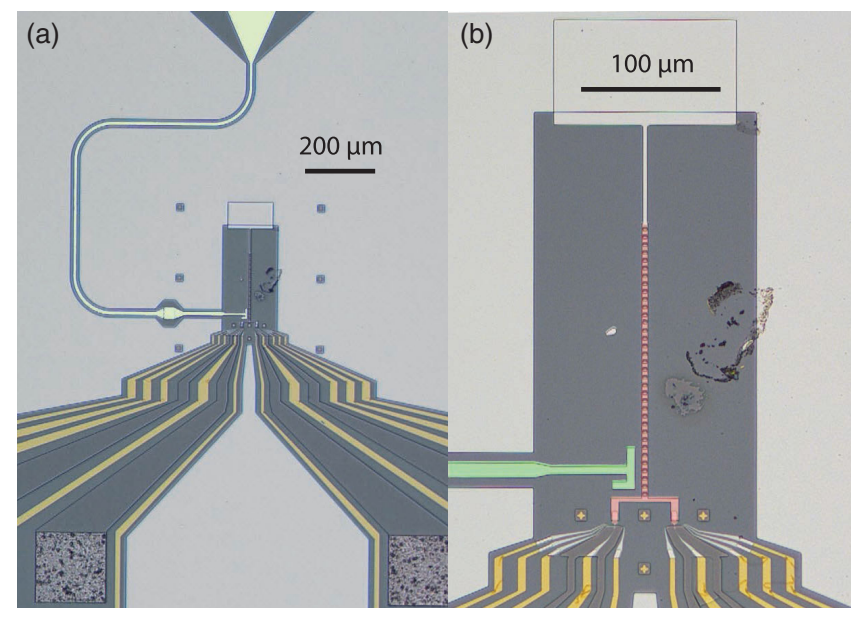

FIG. 5. Overview images of the device with only the drive line (green) and SQUID array resonator (red) false colored. (a) Overview optical image of the device, showing at the top the launcher of the drive line and its waveguide (green). At the bottom, the gate lines (yellow) are visible. The two squares at the bottom left and right are the Ohmic contacts to the 2DEG. (b) Enlarged view of (a).

structures in Figs. 1(b) and 1(c)] are patterned with optical lithography, and $\mathrm{Ti} / \mathrm{Au}(5 / 80 \mathrm{~nm})$ is deposited by electron beam evaporation and then lifted off. At this step, the markers for the electron beam patterning, the gold (yellow) crosses, visible in Figs. 1(c) and 5, are also deposited. The finer structures of the gates are done in a subsequent step.

The ground plane is defined in the last step of optical lithography. The drive line is patterned in this step up to a distance of $200 \mu \mathrm{m}$ from the resonator; see Fig. 5(a). The ground plane is made of Ti/Al $(3 / 200 \mathrm{~nm})$ by lift-off and is deposited by electron beam evaporation. A part of the ground plane, the light gray areas, is visible in Fig. 5.

The first electron beam lithography step defines the fine gates in a PMMA mask for lift-off, using $3 / 25 \mathrm{~nm}$ (Ti/Al) deposited by electron beam evaporation. The resulting fine gates are shown in Fig. 1(e).

In the final step, a PMMA/MMA bilayer resist is patterned with electron beam lithography. The Dolanbridge technique [38] - the angle evaporation of two $\mathrm{Al}$ layers $(35 / 110 \mathrm{~nm})$ interrupted by an oxidation step-is used to create the Josephson junctions for the SQUID array resonator, which is connected to the ground plane and plunger gate of both DQDs. In addition, the resonator drive line is deposited in the same step to assure good alignment between the drive line and the resonator defining the coupling capacitance and, thus, the coupling rate $\kappa_{\text {ext }}$. The drive line splits the ground plane, which is reconnected by multiple wire bonds (not shown in Fig. 5).

The device is bonded in a printed circuit board (PCB) and mounted in a Oxford Triton 200 cryofree dilution refrigerator at the base plate with a typical temperature of approximately $20 \mathrm{mK}$ [39]. 


\section{APPENDIX B: MEASUREMENT SETUP}

The SQUID array resonator is measured in reflection by applying a microwave tone at the drive line (green in Fig. 5). The microwave tone is generated at room temperature and is attenuated $(-20 \mathrm{~dB})$ at the $4 \mathrm{~K}, 100 \mathrm{mK}$, and $20 \mathrm{mK}$ stages before passing through a circulator, which routes it to the resonator and routes the reflected signal to the output line. In the output line, the reflected signal is amplified using a low-noise factory HEMT $(+39 \mathrm{~dB})$ at $4 \mathrm{~K}$ and two amplifiers (+33 dB each) at room temperature, before it is down-converted to an intermediated frequency (IF) of $250 \mathrm{MHz}$. With $+29 \mathrm{~dB}$ amplification, the IF signal is acquired at $1 \mathrm{Gs} / \mathrm{s}$ using an Acqiris U1084A PCIe 8-bit high-speed digitizer.

The dc voltages to the gates are supplied by Yokogawa $7651 \mathrm{dc}$ programmable sources with a 1:11 voltage divider

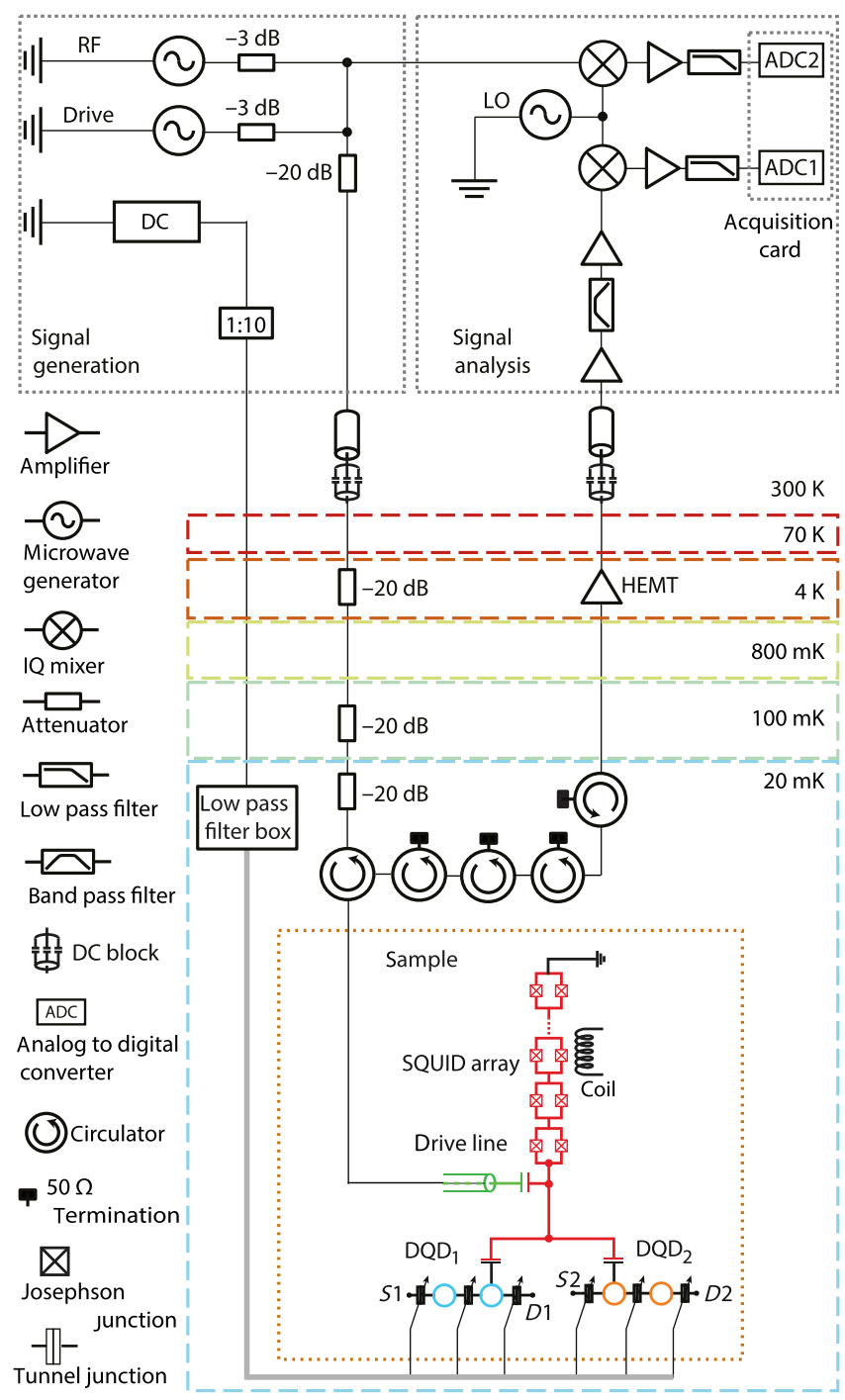

FIG. 6. Simplified schematic of the cryogenic and roomtemperature components and equipment used in the experiments; further details are provided in the text. also acting as a low-pass filter (1 Hz cutoff). The source and drain of both DQDs were grounded in the experiment. At the base temperature, two-stage $R C$ filters with 16 and $160 \mathrm{kHz}$ cutoffs are used at the input of shielded lines leading to the sample holder. All basic characterizations are performed either in direct transport measurements or by using the dispersive shift of the resonator to measure charge stability diagrams; i.e., no additional charge detection is required.

A schematic of the complete setup with all important components is displayed in Fig. 6.

\section{APPENDIX C: MODELING THE SYSTEM}

Here, we discuss the theoretical model used for understanding and fitting the experimental data. We describe the system using the Hamiltonian

$$
H_{\mathrm{tot}}=H_{\mathrm{res}}+\sum_{k} H_{k}+\sum_{k} H_{\mathrm{int}, k},
$$

with the resonator Hamiltonian

$$
H_{\mathrm{res}}=\omega_{r} a^{\dagger} a,
$$

the Hamiltonian for the $k$ th DQD $(k=1,2$ for the experiments discussed here)

$$
H_{k}=-\frac{1}{2} \delta_{k} \sigma_{z}+t_{k} \sigma_{x}
$$

and the coupling between resonator and DQDs,

$$
H_{\mathrm{int}, k}=g_{k} \sigma_{z}\left(a^{\dagger}+a\right) .
$$

Here, we use $\hbar=1$ for simplicity. $a\left(a^{\dagger}\right)$ is the bosonic annihilation (creation) operator, $\omega_{r}$ is the resonator angular frequency, and $\sigma_{x, y, z}$ are Pauli matrices. The DQDs are defined through the charge detuning $\delta_{k}$ and their tunnel splitting $2 t_{k}$. The parameter $g_{k}$ is the dipolar coupling strength between the $k$ th DQD and the resonator. Transforming $H_{\text {tot }}$ into the DQD eigenbasis, we find

$$
\begin{aligned}
H= & \omega_{r} a^{\dagger} a-\frac{1}{2} \sum_{k} \omega_{k} \sigma_{z} \\
& +g_{k} \sum_{k}\left(\sin \theta_{k} \sigma_{x}+\cos \theta_{k} \sigma_{z}\right)\left(a^{\dagger}+a\right) \\
\approx & \omega_{r} a^{\dagger} a-\frac{1}{2} \sum_{k} \omega_{k} \sigma_{z}+g_{k} \sum_{k} \sin \theta_{k}\left(\sigma_{-} a^{\dagger}+\sigma_{+} a\right),
\end{aligned}
$$

where we performed a rotating-wave approximation in the last step to arrive at the well-known Tavis-Cummings Hamiltonian. Here, $\omega_{k}=\sqrt{4 t_{k}^{2}+\delta_{k}^{2}}$ and $\tan \theta_{k}=2 t_{k} / \delta_{k}$, so that $\sin \theta_{k}=2 t_{k} / \omega_{k}$ and $\cos \theta_{k}=\delta_{k} / \omega_{k}$. 


\section{Scattering in input-output theory}

To model reflection of an incident signal from the resonator, we use the scattering Lindblad Hamiltonian (SLH) cascaded quantum system formalism [40]. Modeling the cavity as a single-port resonator with output coupling $\kappa_{\text {ext }}$ and cascading in the coherent probe at signal frequency $\omega_{p}$ [41], we find the total SLH Hamiltonian $H_{\mathrm{SLH}}=H_{\mathrm{tot}}+H_{P}$, where the probe term is

$$
H_{P}=\frac{1}{2 i} \sqrt{\kappa_{\mathrm{ext}}}\left(\alpha a^{\dagger}-\alpha^{*} a\right)
$$

with $\alpha$ the input coherent field amplitude and $\kappa_{\text {ext }}$ the radiative coupling to the external waveguide modes. The probe Hamiltonian $H_{P}$ is already written in a frame rotating at the signal frequency $\omega_{p}$. We transform $H_{\text {tot }}$ into the same rotating frame and find

$$
\begin{aligned}
H_{\mathrm{tot}}= & \delta \omega_{r} a^{\dagger} a-\frac{1}{2} \sum_{k} \delta \omega_{k} \sigma_{z}^{(k)} \\
& +g_{k} \sum_{k} \sin \theta_{k}\left(\sigma_{-}^{(k)} a^{\dagger}+\sigma_{+}^{(k)} a\right),
\end{aligned}
$$

with the detunings $\delta \omega_{r}=\omega_{r}-\omega_{p}$ and $\delta \omega_{k}=\omega_{k}-\omega_{p}$. Including incoherent processes, the time evolution of the systems density matrix $\rho$ follows the master equation

$$
\dot{\rho}=-i\left[H_{\mathrm{SLH}}, \rho\right]+\mathcal{L}_{\mathrm{nr}} \rho+\mathcal{L}_{\mathrm{SLH}} \rho .
$$

The second term on the right-hand side of the master equation (C8) describes all nonradiative losses and dephasing processes. For zero-temperature quantum baths coupled to each quantum dot and the resonator independently, we write this as

$$
\mathcal{L}_{\mathrm{nr}} \rho=\sum_{k} \gamma_{1, k} \mathcal{D}\left[\sigma_{-}\right] \rho+\frac{1}{2} \sum_{k} \gamma_{\varphi, k} \mathcal{D}\left[\sigma_{z}\right] \rho+\kappa_{\mathrm{in}} \mathcal{D}[a] \rho,
$$

with the DQD relaxation rate $\gamma_{1, k}$, their pure dephasing rates $\gamma_{\varphi, k}$, and the internal resonator decay into nonguided modes $\kappa_{\text {in }}$.

Here, we assume that the main loss channels for the DQDs is a coupling to electromagnetic modes of the environment described by the dipole operator

$$
H_{\mathrm{env}}=\sigma_{z} \sum_{k} \beta_{k}\left(b_{k}+b_{k}^{\dagger}\right),
$$

where $b_{k}\left(b_{k}^{\dagger}\right)$ are bosonic annihilation (creation) operators for a mode of the electromagnetic environment to DQD- $k$. Making a Markov approximation, we can find the DQD relaxation and dephasing rates [42]

$$
\begin{aligned}
& \gamma_{1, k}=\sin ^{2} \theta_{k} C\left(\omega_{k}\right), \\
& \gamma_{\varphi, k}=\cos ^{2} \theta_{k} C(0),
\end{aligned}
$$

where $C\left(\omega_{k}\right)$ is the environmental spectral function $C(\omega)=\int d t e^{-i \omega t}\langle\hat{X}(t) \hat{X}(0)\rangle$, with $\hat{X}=\sum_{k} \beta_{k}\left(b_{k}+b_{k}^{\dagger}\right)$. In our calculations, we assume white noise spectra for the noise acting on the DQDs for simplicity.

Finally, the third term on the right-hand side of Eq. (C8) describes the scattering of the input drive fields into the waveguide modes as

$$
\mathcal{L}_{\mathrm{SLH}} \rho=\mathcal{D}[L] \rho,
$$

where

$$
L=\sqrt{\kappa_{\mathrm{ext}}} a+\alpha \mathbb{1} .
$$

We calculate the amplitudes $\beta$ and photon fluxes $n$ of the scattered fields from

$$
\beta=\operatorname{Tr}\{L \rho\}, \quad n=\operatorname{Tr}\left\{L^{\dagger} L \rho\right\},
$$

where $\rho$ is the solution of the master equation (C8). For spectroscopy experiments, as modeled here, it is sufficient to calculate the steady-state scattering, considering $\dot{\rho}=0$.

\section{Two-tone spectroscopy}

In principle, the technique described here would allow us to simulate circuit QED spectroscopy [32] directly, either by adding another set of input and output modes at different frequencies or, assuming that the input at or close to the DQD resonance is not monitored, by adding a coherent drive term to the Hamiltonian. Since there are now multiple time-dependent terms in the Hamiltonian, which oscillate at different frequencies, a single rotating frame is no longer sufficient to capture the dynamics. Instead, one can move towards a multi-tone Floquet analysis or alternatively perform time-dependent simulations of the dynamics to find the response of the system.

In practice, this analysis has proven not feasible, as the number of unknown parameters is too large for reliable fits to the data. We have therefore fitted the qubit spectroscopy experiments with simulations of standard single-tone spectroscopy in the far-detuned regime, adding an additional scale and offset parameter to match the amplitude of the experimental results. We stress that the relative height of the resonances is extracted directly from the master equation; see, e.g., Figs. 10(d) and 10(h). Since the two-tone experiments are in the linear response regime of the resonator phase (weak drive and readout power), i.e., the change in the signal phase is linear in the excitation probability of the DQDs, this technique can still produce quantitative agreement with the experimental data. 


\section{Eigenstates in the coupled system}

To clarify the composition of the eigenstates at the points of maximal coupling, we present here the exact expressions for the two cases where

(a) both DQDs and the resonator are resonant, $\Delta_{r}=\omega_{r}-\omega_{k}=0$, relevant for Fig. 2 of the main text, and

(b) the two DQDs are resonant with each other and the resonator is detuned, $\Delta_{r} \gg g_{k}$, relevant for Figs. 3 and 4 of the main text.

In case (a), the Hamiltonian in the one-excitation subspace can be written as

$$
H_{(a)}=\left(\begin{array}{ccc}
0 & g_{1} & 0 \\
g_{1} & 0 & g_{2} \\
0 & g_{2} & 0
\end{array}\right),
$$

where we subtracted a constant energy offset, $\omega_{k}=\omega_{r}$, and we are considering the basis $\{|e, g, 0\rangle,|g, g, 1\rangle,|g, e, 0\rangle\}$. Diagonalizing this Hamiltonian leads to the eigenstates and eigenenergies

$$
E_{0}=0, \quad E_{-}=-g_{c}, \quad E_{+}=+g_{c}
$$

and corresponding eigenstates
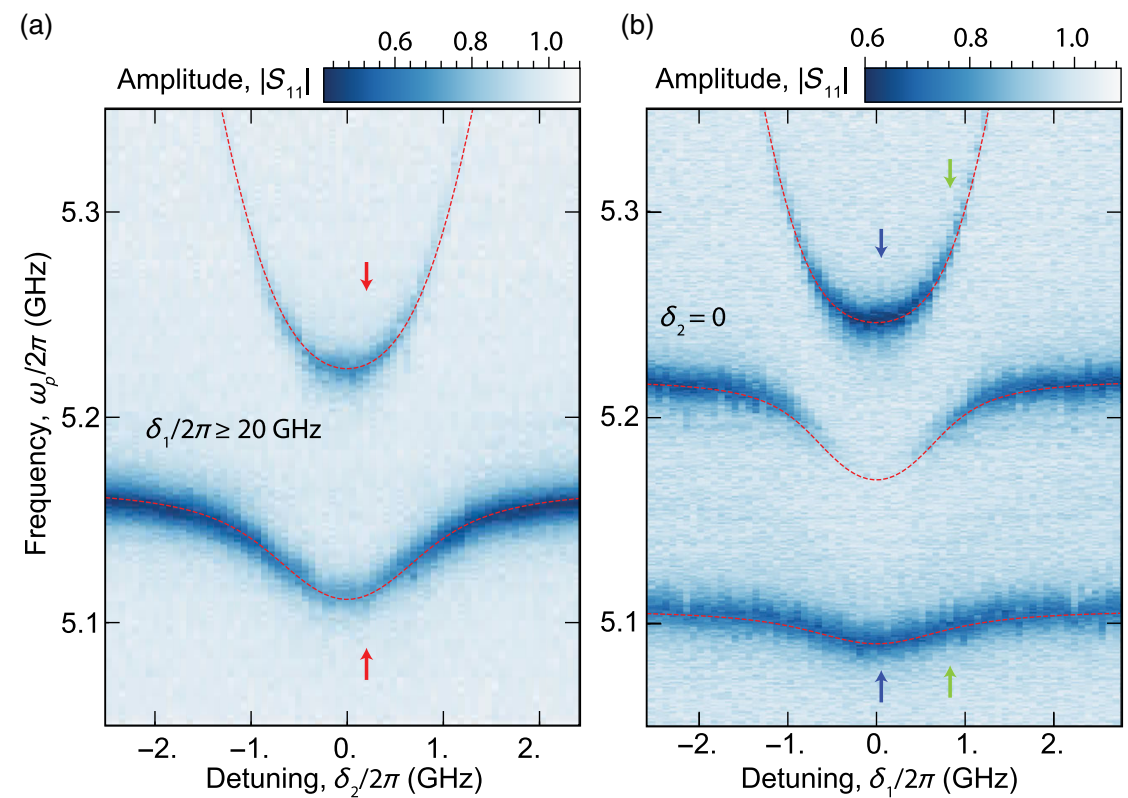

$$
\begin{aligned}
|0\rangle_{r 3} & =\frac{1}{g_{c}}\left(g_{1}|g, e, 0\rangle-g_{2}|e, g, 0\rangle\right), \\
|-\rangle_{r 3} & =\frac{1}{\sqrt{2} g_{c}}\left(g_{2}|g, e, 0\rangle+g_{1}|e, g, 0\rangle-g_{c}|g, g, 1\rangle\right), \\
|+\rangle_{r 3} & =\frac{1}{\sqrt{2} g_{c}}\left(g_{2}|g, e, 0\rangle+g_{1}|e, g, 0\rangle+g_{c}|g, g, 1\rangle\right),
\end{aligned}
$$

with $g_{c}=\sqrt{g_{1}^{2}+g_{2}^{2}}$. Here, the state $|0\rangle_{r 3}$ is a dark state with respect to the coupling to the resonator, as it is an antisymmetric state and the coupling between DQDs and resonator is symmetric, since both DQDs couple to the same phase of the drive field at one end of the resonator. Condition (a) is exactly met when $\omega_{r}=\omega_{\mathrm{DQD} 1}=\omega_{\mathrm{DQD} 2}$, as illustrated by the data in Figs. 2(b) and 2(c), 7(b), 7(e), 7(g), 7(j), 8(b), and 8(e).

The second case (b) we treat here in two equivalent ways. First, we write the Hamiltonian

$$
H_{(b 1)}=\left(\begin{array}{ccc}
0 & g_{1} & 0 \\
g_{1} & \Delta_{r} & g_{2} \\
0 & g_{2} & 0
\end{array}\right),
$$

where the only difference to $H_{(a)}$ is the nonzero energy of the resonator state compared to the DQD states, with $\Delta_{r}=\omega_{r}-\omega_{k} \neq 0$ and $\omega_{k}=0$. Directly diagonalizing this Hamiltonian is possible, but the expressions for the

FIG. 7. Resonator spectroscopy with one or two DQDs tuned into resonance with the resonator. The same charge configuration is used for the data in Fig. 2 of the main text. (a) Tuning $\delta_{2}$ of $\mathrm{DQD}_{2}\left(2 t_{2} / 2 \pi=5.168 \mathrm{GHz}\right)$ into resonance with the SQUID array resonator, when $\mathrm{DQD}_{1}$ is tuned in Coulomb blockade, $\delta_{1} / 2 \pi \gtrsim 20 \mathrm{GHz}$. (b) With $\omega_{r}=\omega_{\mathrm{DQD} 2}, \mathrm{DQD}_{1}\left(2 t_{1} / 2 \pi=5.183 \mathrm{GHz}\right)$ is brought in resonance by changing $\delta_{1}$. (c)-(e) are line traces of (a) and (b) to show the conditions $\omega_{r}=\omega_{\mathrm{DQD} 2}$ (red arrows), $\omega_{\mathrm{DQD} 2}+g_{1} \approx \omega_{\mathrm{DQD} 1}$ (green arrows), and $\omega_{r}=\omega_{\mathrm{DQD} 2}=\omega_{\mathrm{DQD} 1}$ (blue arrows). All fits in (c)-(e) are fits to the master equation with extracted parameters listed and discussed in Appendix H. 

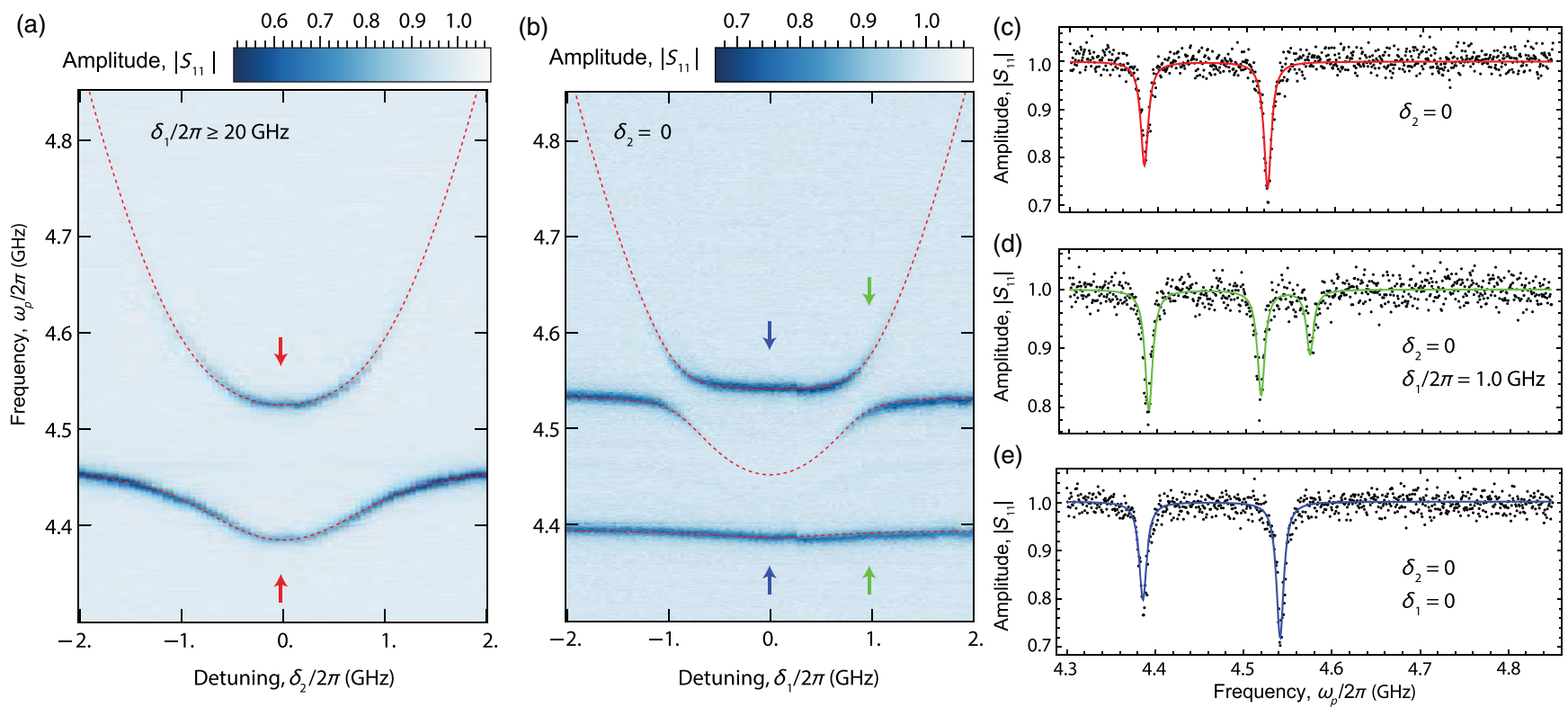

(f)

(g)
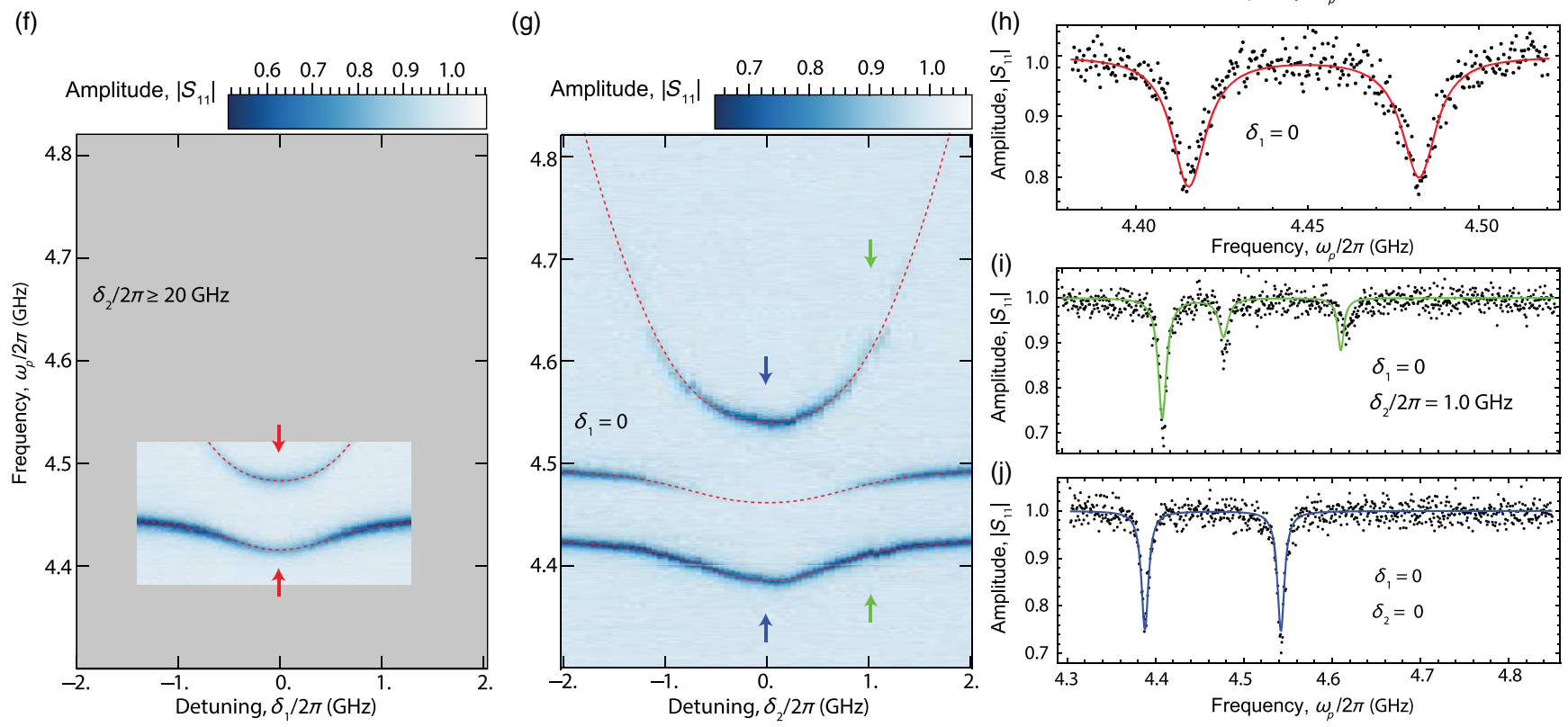

FIG. 8. Resonator spectroscopy with one or two qubits tuned into resonance with the resonator. Similar to the data in Fig. 2 except that the device is biased at a point with coupling rates $g_{1} / 2 \pi=34 \mathrm{MHz}$ and $g_{2} / 2 \pi=69 \mathrm{MHz}$. All data are fitted with master-equation simulations (Appendixes $\mathrm{C}$ and $\mathrm{H}$ ). (a) Measuring the reflection spectrum $\left|S_{11}\right|$ while tuning $\delta_{2}$ of $\mathrm{DQD}_{2}\left(2 t_{2} / 2 \pi=4.448 \mathrm{GHz}\right)$ and DQD 1 is in Coulomb blockade $\delta_{1} / 2 \pi \gtrsim 20 \mathrm{GHz}$. The resonator is flux tuned to $\omega_{r} / 2 \pi=4.462 \mathrm{GHz}$. (b) Reflection spectrum $\left|S_{11}\right|$, when resonator $\mathrm{DQD}_{2}$ are in resonance $\left(\delta_{2}=0\right)$, the detuning $\delta_{1}$ of the weaker coupled DQD $\left(2 t_{1} / 2 \pi=4.452 \mathrm{GHz}\right)$ is swept. (c) Line trace of reflection spectrum $\left|S_{11}\right|$ in (a) at the red arrows showing two separate resonances. (d) Resonator spectroscopy line trace obtained from (b) when $\omega_{\mathrm{DQD} 2}+g_{2} \approx \omega_{\mathrm{DQD} 1}$ (green arrows). (e) Resonator spectroscopy line trace adopted from (b) at $\delta_{1,2}=0$ obtaining $\omega_{r}=\omega_{\mathrm{DQD} 1}=\omega_{\mathrm{DQD} 2}$ (blue arrows). (f) Reflection spectrum $\left|S_{11}\right|$, while tuning $\delta_{1}$ of $\mathrm{DQD}_{1}\left(2 t_{1} / 2 \pi=4.450 \mathrm{GHz}\right)$, when $\mathrm{DQD}_{2}$ is in Coulomb blockade $\delta_{2} / 2 \pi \gtrsim 20 \mathrm{GHz}$. (g) Keeping DQD $\mathrm{D}_{1}$ resonator in resonance, $\mathrm{DQD}_{2}\left(2 t_{2} / 2 \pi=4.461 \mathrm{GHz}\right)$ is tuned vs $\delta_{2}$, resulting in hybridization in the case where all three systems are in resonance, $\omega_{r}=\omega_{\mathrm{DQD} 2}=\omega_{\mathrm{DQD} 1}$. (h) Line trace of (f) (red arrows) showing the reflection spectrum $\left|S_{11}\right|$ with two resonances. (i) Line trace of $(\mathrm{g})$ (green arrows) taken at $\omega_{\mathrm{DQD} 1}+g_{1} \approx \omega_{\mathrm{DQD} 2}$, showing the $\mathrm{DQD}_{2}$ tuned into resonance with the hybridized $\mathrm{DQD}_{1}$ resonator state, $|+\rangle_{r 3}$. (j) Data taken at the similar configuration as (e), $\delta_{1,2}=0$, line trace of the $\left|S_{11}\right|$ in (g) (blue arrows).

eigenstates do not lend themselves to quick insights. Instead, we assume the relevant limit $\Delta_{r} \gg g_{1}, g_{2}$, so that we can approximate $\sqrt{\Delta_{r}^{2}+4 g_{c}^{2}} \approx \Delta_{r}\left\{1+\left[\left(2 g_{c}^{2}\right) /\left(\Delta_{r}^{2}\right)\right]\right\}$ and find in this limit

$$
E_{+}^{\prime}=0, \quad E_{-}^{\prime}=-\frac{g_{c}^{2}}{\Delta_{r}}, \quad E_{1}^{\prime}=\Delta_{r}+\frac{g_{c}^{2}}{\Delta_{r}}
$$

with the corresponding (unnormalized) eigenstates 


$$
\begin{aligned}
\left|+^{\prime}\right\rangle_{r 3}= & \frac{1}{g_{c}}\left(g_{1}|g, e, 0\rangle-g_{2}|e, g, 0\rangle\right) \\
\left|-^{\prime}\right\rangle_{r 3}= & \frac{1}{g_{c} \sqrt{g_{c}^{2}+\Delta_{r}^{2}}} \\
& \times\left(g_{2} \Delta_{r}|g, e, 0\rangle+g_{1} \Delta_{r}|e, g, 0\rangle-g_{c}^{2}|g, g, 1\rangle\right) \\
\approx & \frac{1}{g_{c}}\left(g_{2}|g, e, 0\rangle+g_{1}|e, g, 0\rangle-\frac{g_{c}^{2}}{\Delta_{r}}|g, g, 1\rangle\right) \\
\left|1^{\prime}\right\rangle_{r 3}= & \frac{1}{\sqrt{g_{c}^{2}+\Delta_{r}^{2}}}\left(g_{2}|g, e, 0\rangle+g_{1}|e, g, 0\rangle+\Delta_{r}|g, g, 1\rangle\right) \\
\approx & \frac{1}{\Delta_{r}}\left(g_{2}|g, e, 0\rangle+g_{1}|e, g, 0\rangle+\Delta_{r}|g, g, 1\rangle\right)
\end{aligned}
$$

with $g_{c}=\sqrt{g_{1}^{2}+g_{2}^{2}}$. The states $\left| \pm^{\prime}\right\rangle_{r 3}$ are the qubitlike states and $\left|1^{\prime}\right\rangle_{r 3}$ is the resonatorlike state used in the energy diagram in Fig. 3(c). The difference in visibility of the $| \pm\rangle_{d 2}$ states in Fig. 4(a) when changing the resonator detuning $\Delta_{r}$ is fully captured in this approximation. The last term in $\left|-^{\prime}\right\rangle_{r 3}$ contains the excited state of the resonator, and its coefficient is proportional to $1 / \Delta_{r}$ so that, if we increase the resonator detuning, this coefficient and the visibility in spectroscopy decreases. As is observed in Fig. 4(a), the visibility of the darker state $|+\rangle_{r 3}$ remains constant, while the brighter state $|-\rangle_{r 3}$ becomes weaker.

In the same spirit, we may take the coupling $g_{k}$ as a perturbation and, starting from the Hamiltonian Eq. (C16), find the approximate Hamiltonian for the DQDs in perturbation theory up to second order in $g_{k} / \Delta_{r}$ as

$$
H_{(b 2)}=\frac{1}{\Delta_{r}}\left(\begin{array}{cc}
g_{1}^{2} & g_{1} g_{2} \\
g_{1} g_{2} & g_{2}^{2}
\end{array}\right),
$$

in the basis $\{|e, g\rangle,|g, e\rangle\}$. Note that here the two states are not in resonance, due to each state's second-order energy correction obtained from the resonator dispersive shift, which assumes that the DQDs are tuned such that, in the absence of the resonator, they would be resonant with each other. The eigenvalues and eigenstates of this perturbative Hamiltonian are

$$
\begin{aligned}
& \bar{E}_{+}=0, \quad|+\rangle_{d 2}=\frac{1}{g_{c}}\left(g_{1}|g, e\rangle-g_{2}|e, g\rangle\right), \\
& \bar{E}_{-}=-\frac{g_{c}^{2}}{\Delta_{r}}, \quad|-\rangle_{d 2}=\frac{1}{g_{c}}\left(g_{2}|g, e\rangle+g_{1}|e, g\rangle\right),
\end{aligned}
$$

which is identical to Eq. (C18) in the limit $\Delta_{r} \gg g_{k}$.

In Fig. 4(a) of the main text, the coherent qubit-qubit exchange interaction as a function of the detuning from the resonator $\Delta_{r}$ is investigated. The dark state $|+\rangle_{d 2}$ remains at a fixed transition frequency, as is observed in the experiment indicated by the blue line in Fig. 4(a). In Eq. (C20), the energy $\bar{E}_{+}$is constant, and it is the bright state $|-\rangle_{d 2}$ which shifts in energy as a function of $\Delta_{r}$.

Note that the splitting between eigenstates in the case treated here is different from the standard case treated most commonly in the literature, when assuming the DQDs are resonant and coupled through a second-order transition via the resonator. In that case, the diagonal terms in Eq. (C19) would be equal, corresponding to a tuning point where the dressed frequencies of both DQDs are resonant. In that case, we find a splitting of $2 J=2 g_{1} g_{2} / \Delta_{r}$ instead of the value obtained here $\left(g_{1}^{2}+g_{2}^{2}\right) / \Delta_{r}$. The two cases are equivalent only for equal coupling $g_{1}=g_{2}$. Indeed, for the data shown in Fig. 4 , we use $2 J=2 g_{1} g_{2} / \Delta_{r}$, since we have access only to the dispersively shifted frequencies of the DQDs in the measurements.

\section{APPENDIX D: COMPLEMENTARY DATA TO FIG. 2}

In addition to the data in the main text (Fig. 2), we show here the vacuum Rabi mode splitting of $\mathrm{DQD}_{2}$ with the resonator; see Figs. 7(a) and 7(c). We find with our master-equation fitting that $\omega_{r}=\omega_{\mathrm{DQD} 2}$ is realized at $\delta_{2} / 2 \pi=0.2 \mathrm{GHz}$. This bias point is used to extract the coupling rate from the resonator to $\mathrm{DQD}_{2}, g_{2} / 2 \pi=$ $56 \mathrm{MHz}$ [Fig. 7(c)], and is quoted in the main text. The collective mode coupling, realized by tuning $\mathrm{DQD}_{1}$ $\left(g_{1} / 2 \pi=53 \mathrm{MHz}\right)$ into resonance when $\mathrm{DQD}_{2}$ is in vacuum Rabi mode splitting, to obtain the resonant condition $\omega_{r}=\omega_{\mathrm{DQD} 2}=\omega_{\mathrm{DQD} 1}$, which is essentially the same bias point as Fig. 2(c), indicates that the DQD system is fully tunable via the parameters $2 t_{1,2}$ and $\delta_{1,2}$, allowing us to measure data equivalent to that shown in Fig. 2.

\section{APPENDIX E: RESONANT INTERACTION WITH UNEQUAL COUPLING RATES $g_{1} \neq g_{2}$}

In the main text in Fig. 2 and Appendix D, we present the vacuum Rabi mode splitting and the collective vacuum Rabi mode-splitting measurements when varying the charge detuning of $\mathrm{DQD}_{1}$ and $\mathrm{DQD}_{2}$ for the configuration where both coupling rates are approximately equal, $g_{1,2} / 2 \pi \approx 55 \mathrm{MHz}$. We use a bias point in Figs. 3(b) and 4 , where $g_{1} / 2 \pi=34 \mathrm{MHz}$ and $g_{2} / 2 \pi=69 \mathrm{MHz}$, to measure the $2 J$ splitting of the virtual photon-mediated qubit-qubit exchange interaction. The coupling rates used to fit the data in Figs. 3(b) and 4 are obtained from the vacuum Rabi mode-splitting measurements shown in Fig. 8. See Appendix $\mathrm{H}$ for a detailed discussion of the fitting procedure employed.

In Fig. 8, we present the on-resonance interaction for this configuration similar to Fig. 2. Also here, we observe a dark state when $\omega_{r}=\omega_{\mathrm{DQD} 1}=\omega_{\mathrm{DQD} 2}$, by tuning the detuning parameters $\delta_{1,2}$. In principle, Figs. 8(b) and 8(g) are showing data of very similar experiments, as one DQD is in resonance with the resonator and the opposite DQD is tuned in 
resonance for the data obtained in both panels. For both data sets, when the qubits are at $\delta_{1,2}=0$ [Figs. 8(e) and 8(j)], ideally the two measurements should be identical, but they have been realized in two independent procedures and thus suffer from slightly different resonator responses. The unequal coupling rate to the resonator makes the response of the amplitude of the reflection spectrum $\left|S_{11}\right|$ quite different. This difference is visible by the initial small (large) vacuum Rabi splitting at $\delta_{2,(1)} / 2 \pi=-2 \mathrm{GHz}$ set by the coupling rate $g_{1,(2)}$. The collective mode coupling to the resonator is $g_{c} / 2 \pi=\sqrt{g_{1}^{2}+g_{2}^{2}} / 2 \pi=76 \mathrm{MHz}$.

In addition, we present line traces in Fig. 8 for the vacuum Rabi splitting $\left[\omega_{r}=\omega_{\mathrm{DQD} k}\right.$, Figs. 8(c) and 8(h)], collective mode coupling $\left[\omega_{r}=\omega_{\mathrm{DQD} 1}=\omega_{\mathrm{DQD} 2}\right.$, Figs. $8(\mathrm{e})$ and $\left.(8 \mathrm{j})\right]$, and the case where the $|+\rangle_{r 2}$ state is approximately resonant with the DQD which is being tuned $\left[\omega_{\mathrm{DQD} 2}+g_{1} \approx \omega_{\mathrm{DQD} 2}\right.$ in Fig. 8(d) and $\omega_{\mathrm{DQD} 1}+g_{2} \approx \omega_{\mathrm{DQD1}}$ in Fig. 8(i)]. The latter case shows clearly the difference in coupling strengths to the resonator of both DQDs. This difference is fully captured by our master-equation simulations (solid and dashed lines in Fig. 8).

\section{APPENDIX F: SPECTROSCOPY OF DISPERSIVE QUBIT-QUBIT INTERACTION FOR THE $2 g_{1} \approx g_{2}$ CONFIGURATION}

In Figs. 3(a) and 3(b) of the main text, $\mathrm{DQD}_{1}$ and $\mathrm{DQD}_{2}$ are tuned into resonance, fulfilling the condition $\omega_{\mathrm{DQD} 1}=$ $\omega_{\text {DQD2 }}$ resulting in the hybridized states $| \pm\rangle_{d 2}$. With $\mathrm{DQD}_{2}$ $\left(\mathrm{DQD}_{1}\right.$ ) largely detuned by setting $\delta_{1,2} / 2 \pi \gtrsim 20 \mathrm{GHz}$, we observe the DQD single-charge qubit behavior, since we can fit it by the expected spectrum, $\omega_{\mathrm{DQD}}=\sqrt{4 t^{2}+\delta^{2}}$; see
Fig. 9. This observation demonstrates full gate control of each DQD qubit and excludes coupling to spurious twolevel fluctuators [43]. In addition, we present the specular tuning of the DQDs compared to the data shown in Figs. 3(b) and 9. Here, the DQDs are tuned to $2 t_{1} / 2 \pi=$ $4.453 \mathrm{GHz}$ and $2 t_{2} / 2 \pi=4.472 \mathrm{GHz}, \delta_{2}=0$ realizing resonance $\left(\omega_{\mathrm{DQD} 1}=\omega_{\mathrm{DQD} 2}\right)$ at finite detuning $\left(\delta_{1} / 2 \pi=\right.$ $\pm 0.4 \mathrm{GHz}$ ), showing clear hybridization between the twoqubit state via virtual photon exchange [Fig. 9(c)]. Here, qubit spectroscopy shows different qubit contrast for the two DQDs, which is attributed to the difference in coupling rate $g_{1,2}$. At detuning, $\left|\delta_{1} / 2 \pi\right|= \pm 0.6 \mathrm{GHz}$, indicated by the orange dots in Fig. 9(c), the bare qubit frequencies are equal, resulting in a dark state. The resonance frequencies in Fig. 9(c) are fitted to the full Hamiltonian model for the interacting $\left(g_{k} \neq 0\right.$ red dashed line) and noninteracting case ( $g_{k}=0$ green dashed line), showing quantitative agreement with the data. The fact that the lower red dashed line in Fig. 9(c) does not converge to the lower green dashed line for large $\left|\delta_{1}\right|$ can be attributed to the breakdown of the dispersive approximation in this regime.

The tunnel rates $2 t_{1,2}$, obtained for the data in Figs. 9(a) and 9(b), are not exactly the same as in Figs. 3(b) and 9(c), since these data is measured in a separate run (about one month) later in the same bias configuration.

\section{APPENDIX G: COHERENT QUBIT-QUBIT EXCHANGE INTERACTION IN SPECTROSCOPY WITH EQUAL COUPLING RATE $g_{1} \approx g_{2}$}

In the main text, we use the configuration $g_{1} / 2 \pi=$ $34 \mathrm{MHz}$ and $g_{2} / 2 \pi=69 \mathrm{MHz}$ to demonstrate the coherent qubit-qubit exchange interaction in qubit spectroscopy.
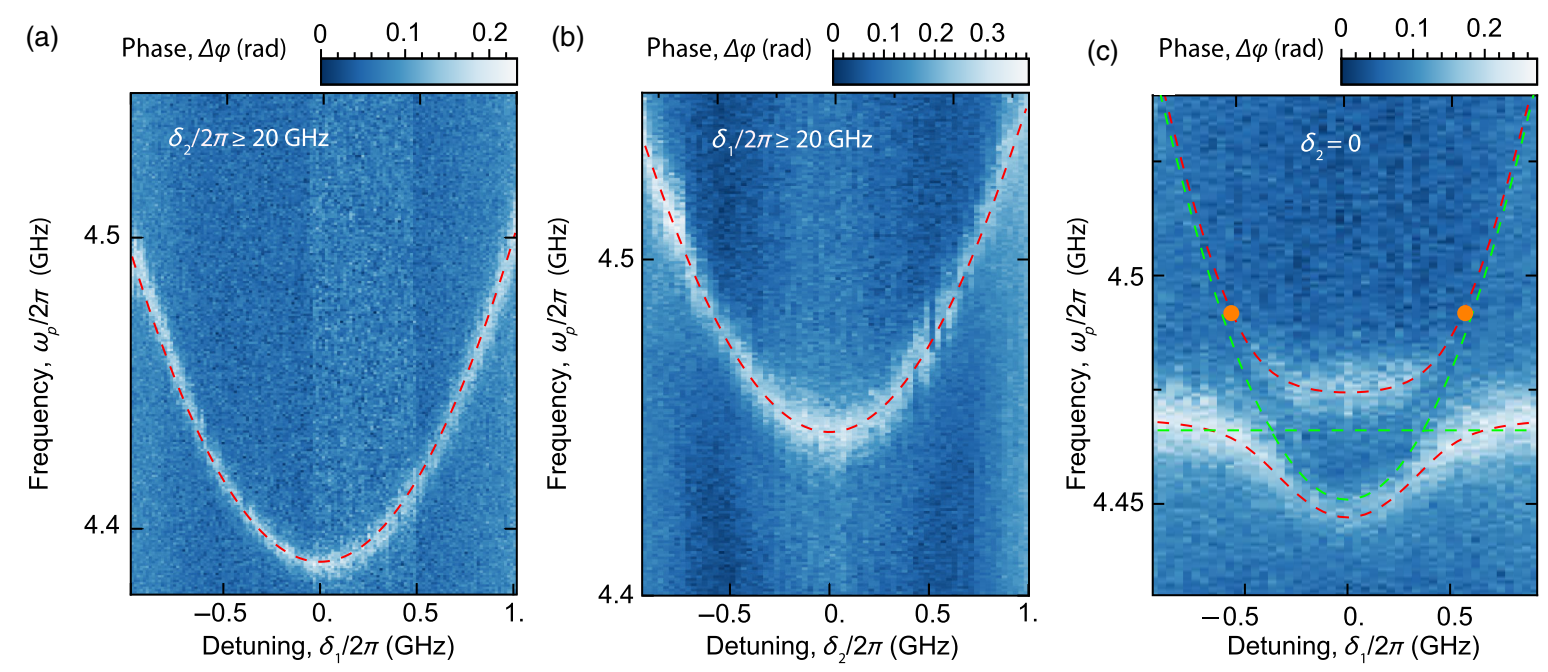

FIG. 9. Supplementary data to Fig. 3(b) showing qubit spectroscopy of the individual DQDs with the resonator tuned to $\omega_{r} / 2 \pi=4.726 \mathrm{GHz}$. Dashed lines are fits to extract relevant parameters. (a) Qubit spectroscopy of $\mathrm{DQD}_{1}\left(2 t_{1} / 2 \pi=4.392 \mathrm{GHz}\right)$ vs detuning $\delta_{1}$ in the dispersive regime. (b) Qubit spectroscopy of $\mathrm{DQD}_{2}\left(2 t_{2} / 2 \pi=4.448 \mathrm{GHz}\right)$ vs detuning $\delta_{2}$ in the dispersive regime. (c) Qubit spectroscopy measuring the resonator phase shift with $\mathrm{DQD}_{2}$ fixed $\left(2 t_{2} / 2 \pi=4.472 \mathrm{GHz}, \delta_{2}=0\right)$ and $\mathrm{DQD}$ $\left(2 t_{1} / 2 \pi=4.453 \mathrm{GHz}\right)$ tuned with $\delta_{1}$. 

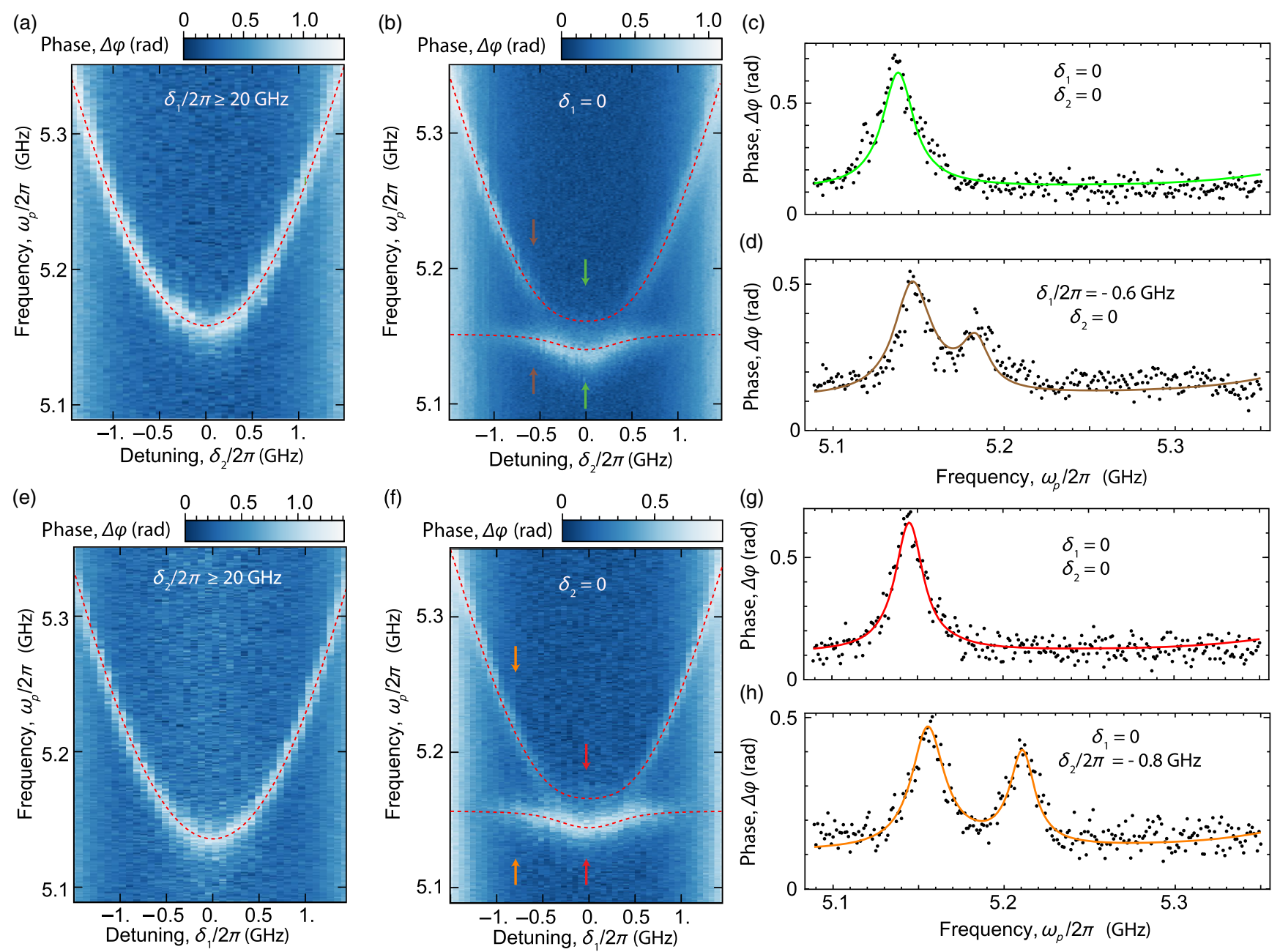

(h)

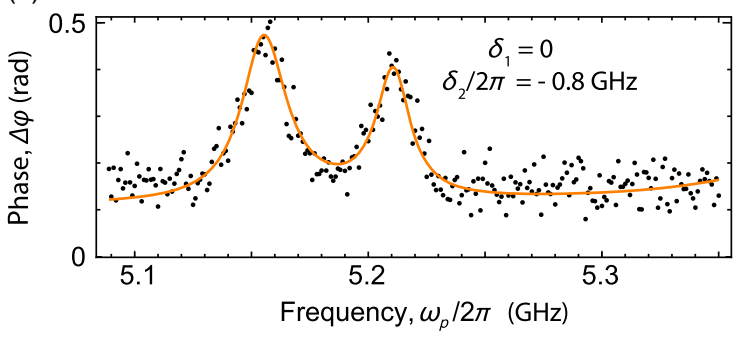

FIG. 10. Single- and two-qubit spectroscopy in the dispersive regime. The readout is performed at $5.454 \mathrm{GHz}$ by populating the resonator with approximately 0.3 photons on average, for all data shown. Red dashed lines are fits to the master-equation simulations, and solid lines are master-equation simulations; see Appendix H. (a) Qubit spectroscopy of $\mathrm{DQD}_{2}\left(2 t_{2} / 2 \pi=5.168 \mathrm{GHz}\right) \mathrm{vs}$ detuning $\delta_{1}$. DQD ${ }_{1}$ is detuned to $\delta_{1} / 2 \pi \gtrsim 20 \mathrm{GHz}$. (b) Qubit spectroscopy with $\mathrm{DQD}_{1}$ at $\delta_{1}=0$ and performing qubit spectroscopy vs $\delta_{2}$. (c) Line trace at $\delta_{1,2}=0$ from (b) (red arrows), obtained at $\omega_{\mathrm{DQD} 1}=\omega_{\mathrm{DQD} 2}$. (d) Line trace of (b) when both DQDs slightly detuned, $\omega_{\mathrm{DQD} 1} \neq \omega_{\mathrm{DQD} 2}$, showing two separate resonances. (e) Qubit spectroscopy of DQD $1\left(2 t_{1} / 2 \pi=5.146 \mathrm{GHz}\right) \mathrm{vs}$ detuning $\delta_{1}$. DQD 2 is detuned to $\delta_{2} / 2 \pi \gtrsim 20 \mathrm{GHz}$. (f) At $\delta_{2}=0$ qubit spectroscopy vs changing detuning $\delta_{1}$. (g) Line trace at $\delta_{1,2}=0$ from (f) (red arrows) showing one resonance peak. (h) Line trace of (f) for detuned DQDs.

At the resonance condition $\omega_{\mathrm{DQD} 1}=\omega_{\mathrm{DQD} 2}$, the $|+\rangle_{d 2}$ state is a dark state for two equally coupled DQDs. We verify this result by using this bias point to demonstrate two-qubit interaction in Fig. 3(a) resulting in a dark state. Here, we present additional data and line traces to support our finding. In Figs. 10(a) and 10(e), we show that both DQDs display the typical level structure of a charge qubit with $\omega_{\mathrm{DQD}}=\sqrt{4 t^{2}+\delta^{2}}$.

Fixing one DQD at zero detuning $\delta=0$ and tuning the other one, we observe virtual photon qubit-qubit exchange interaction resulting in hybridized states. The higherfrequency state $|+\rangle_{d 2}$ is dark, since it is antisymmetric and thus cannot be excited by a symmetric probe to both DQDs with the same phase. Correspondingly, on resonance only single resonances are observed in the line traces in Figs. 10(c) and 10(g). By detuning one of the DQDs, the second resonance can be excited as well again [Figs. 10(d) and 10(h)]. The effect is fully captured by our masterequation simulations (lines in Fig. 10). In the main text, we instead discuss the device tuned to a bias point where $g_{1} \neq g_{2}$; see Figs. 3(b) and 3(c).

\section{APPENDIX H: DESCRIPTION OF FITTING PROCEDURE AND EXTRACTED PARAMETERS}

In this Appendix, we describe the procedure we use for fitting the data and extracting the master-equation parameters. 
In general, if not stated otherwise, fits are least-square fits to the full master-equation input-output model of the system, Eq. (C8). In order to reduce the number of independent fit parameters, we adopt an iterative approach, where we successively extract different fit parameters from different parts of the spectrum.

We illustrate the procedure using the example of Fig. 2 (Table I). The same procedure is followed for the data shown in Fig. 7 (Table II). Initially, we use the leftmost line trace in Fig. 2(a), which is a resonatorlike resonance, to obtain initial estimates for the resonator decay rates $\kappa_{\text {int }}$ and $\kappa_{\text {ext }}$ as well as the resonator frequency $\omega_{r}$. These values are then used as initial parameters to fit the line trace shown in Fig. 2(b), close to resonance between $\mathrm{DQD}_{1}$ and the resonator, $\omega_{r}=\omega_{\mathrm{DQD1}}$. The quoted frequencies in the main text are the measured Lamb-shifted frequencies [32]. From this fit, we extract the DQD coupling strength $g_{1}$ and its linewidth $\gamma_{2,1}^{*}=$ $\gamma_{1,1} / 2+\gamma_{2,1}$; see Table I. Note that in all the fits we set the pure dephasing for each DQD to zero, $\gamma_{2, k}=0$, as its effect on the scattering spectrum is essentially indistinguishable from the relaxation rates $\gamma_{1, k}$. The essence of the fits is to capture the linewidth of the resonances, given by $\gamma_{2,1}^{*}$. In principle, taking into account the line traces at finite detuning $(\delta \neq 0)$ could additionally provide insight into the relaxation and dephasing rates. As here this insight is not essential to obtain more accurate fits, we decide to keep $\gamma_{2, k}$ fixed for simplicity. The extracted DQD linewidth $\gamma_{2, k}$ is measured at finite power and is close to the extrapolated zero power limit $\Gamma_{2,1} / 2 \pi=4.8 \pm 0.6 \mathrm{MHz}$, measured independently in the dispersive regime (see the main text). We generally observe that the extracted qubit linewidths are power broadened, as the values are typically $1-3 \mathrm{MHz}$ higher than $\Gamma_{2,1} / 2 \pi$ compared, for example, with the values in Table I.

We also extract the resonator internal and external loss rates from independent measurements (not shown) with both DQDs detuned $\left(\delta_{1,2} / 2 \pi \gtrsim 20 \mathrm{GHz}\right)$ and find

TABLE I. Extracted values from the data shown in Fig. 2 by fitting the line traces as described in the text. For Figs. 2(a) and 2(b), the line trace at $\delta_{1}=0$ is used. We extract the parameters of the data presented in Fig. 2(d) at detuning $\delta_{2} / 2 \pi=-2.9 \mathrm{GHz}$. The extracted parameters in column Fig. 2(c) are those of $\mathrm{DQD}_{2}$ with the resonator and $\mathrm{DQD}_{1}$ parameters fixed (indicated by ${ }^{*}$ ).

\begin{tabular}{lccc}
\hline \hline & $\begin{array}{c}\text { Figures 2(a) } \\
\text { and 2(b) }\end{array}$ & Figure 2(d) & Figure 2(c) \\
\hline$\omega_{r} / 2 \pi(\mathrm{MHz})$ & $5170 \pm 1$ & $5172 \pm 1$ & $5172^{*}$ \\
$\kappa_{\text {int }} / 2 \pi(\mathrm{MHz})$ & $18 \pm 2$ & $17 \pm 1$ & $17^{*}$ \\
$\kappa_{\text {ext }} / 2 \pi(\mathrm{MHz})$ & $6.5 \pm 0.1$ & $6.1 \pm 0.1$ & $6.1^{*}$ \\
$2 t_{1} / 2 \pi(\mathrm{MHz})$ & $5166 \pm 1$ & $5138 \pm 1$ & $5138^{*}$ \\
$g_{1} / 2 \pi(\mathrm{MHz})$ & $53.4 \pm 0.2$ & $51.1 \pm 0.4$ & $51.1^{*}$ \\
$\gamma_{2,1}^{*} / 2 \pi(\mathrm{MHz})$ & $5.3 \pm 0.9$ & $6.4 \pm 1.2$ & $6.4^{*}$ \\
$2 t_{2} / 2 \pi(\mathrm{MHz})$ & $\ldots$ & $\ldots$ & $5156.2 \pm 0.6$ \\
$g_{2} / 2 \pi(\mathrm{MHz})$ & $\ldots$ & $\ldots$ & $56.7 \pm 0.2$ \\
$\gamma_{2,2}^{*} / 2 \pi(\mathrm{MHz})$ & $\ldots$ & $\ldots$ & $6.0 \pm 0.6$ \\
\hline \hline
\end{tabular}

TABLE II. Extracted values from the data shown in Fig. 7 by fitting the line traces as described in the text. For Figs. 7(a) and 7 (c), the line trace at $\delta_{2} / 2 \pi=0.2 \mathrm{GHz}$ is used. For extracting the values indicated as Fig. 7(b), we use the data in this figure at detuning $\delta_{1} / 2 \pi=-2.9 \mathrm{GHz}$, leftmost line trace. The extracted parameters indicated as Fig. 7(e) are used to extract the $\mathrm{DQD}_{1}$ parameters with the resonator and $\mathrm{DQD}_{2}$ parameters fixed (indicated by *).

\begin{tabular}{lccc}
\hline \hline & $\begin{array}{c}\text { Figures 7(a) } \\
\text { and 7(c) }\end{array}$ & Figure 7(b) & Figure 7(e) \\
\hline$\omega_{r} / 2 \pi(\mathrm{MHz})$ & $5170.9 \pm 0.8$ & $5167.8 \pm 0.9$ & $5167.8^{*}$ \\
$\kappa_{\text {int }} / 2 \pi(\mathrm{MHz})$ & $12 \pm 2$ & $10 \pm 2$ & $10^{*}$ \\
$\kappa_{\text {ext }} / 2 \pi(\mathrm{MHz})$ & $5.7 \pm 0.1$ & $5.7 \pm 0.1$ & $5.7^{*}$ \\
$2 t_{2} / 2 \pi(\mathrm{MHz})$ & $5167.9 \pm 0.8$ & $5157.3 \pm 0.8$ & $5157.3^{*}$ \\
$g_{2} / 2 \pi(\mathrm{MHz})$ & $55.9 \pm 0.3$ & $55.8 \pm 0.3$ & $55.8^{*}$ \\
$\gamma_{2,2}^{*} / 2 \pi(\mathrm{MHz})$ & $11 \pm 2$ & $12 \pm 2$ & $12^{*}$ \\
$2 t_{1} / 2 \pi(\mathrm{MHz})$ & $\ldots$ & $\ldots$ & $5183.1 \pm 0.7$ \\
$g_{1} / 2 \pi(\mathrm{MHz})$ & $\ldots$ & $\ldots$ & $53.4 \pm 0.3$ \\
$\gamma_{2,1}^{*} / 2 \pi(\mathrm{MHz})$ & $\ldots$ & $\ldots$ & $5.7 \pm 0.6$ \\
\hline \hline
\end{tabular}

$\kappa_{\text {int }} / 2 \pi=18.7 \pm 0.5 \mathrm{MHz}$ and $\kappa_{\text {ext }} / 2 \pi=7.4 \pm 0.2 \mathrm{MHz}$ for $\omega_{r} / 2 \pi=5.170 \mathrm{GHz}$, which is comparable to what is obtained from the data in Fig. 2 and listed in Table I. Finally, to calibrate the detuning axis, we perform a simultaneous fit to three different line traces (not shown) in Fig. 2(a), using the parameters from the previous fit.

Even though the gate settings are exactly the same for $\mathrm{DQD}_{1}$ in the measurements shown in Figs. 2(b) and 2(d) as the later one measured 2 days later, a small frequency shift $(30 \mathrm{MHz})$ of the DQD tunnel rate is extracted from the fit. We attribute this shift to changes in the environmental offset charge distribution, influencing the effective applied gate voltages, which effectively shift the tunnel rate $2 t_{1}$. The shift is small but has to be taken into account to improve the quality of the fits; compare the values in Table I. Fitting to the data in Fig. 2(d), we start with a single line trace at large negative detuning $\left(\delta_{2} / 2 \pi=\right.$ $-2.9 \mathrm{GHz}$ ), where the resonator and $\mathrm{DQD}_{1}$ are resonant. We fit the resonator and $\mathrm{DQD}_{1}$ parameters to this line trace and use those parameters as fixed (indicated by $*$ in Table I) when obtaining the parameters for $\mathrm{DQD}_{2}$ from a fit at $\delta_{2}=0$, where all three systems are close to resonant. The extracted parameters are displayed in Table I.

To obtain the parameter values for the data presented in Fig. 8, the procedure is modified slightly. We first extract resonance positions from the experimental data using a simple fit to Lorentzians and then use a pure Hamiltonian model, Eq. (C5), to fit all the Hamiltonian parameters to the spectrum. Then, we apply the master-equation simulation with these parameters as input to single line traces of the data to obtain the linewidths. All parameters extracted from the data presented in Fig. 8 are displayed in Table III.

With this procedure, the agreement between the theory and experiment is not quite as good as for the full 
TABLE III. Extracted values from the data shown in Fig. 8 by fitting the line traces as described in the text. For the parameters indicated by Figs. 8(a) and 8(b), a Hamiltonian fit to the resonance positions is performed. For the parameter indicated with Figs. 8(c)-8(e), a master-equation fit is performed by fixing the parameters obtained from the previous fit (indicated by $*$ ). For the parameters indicated by Figs. 8(f) and 8(g), a Hamiltonian fit to the resonance positions is performed. For the parameter indicated with Figs. 8(i)-8(k), a master-equation fit is performed by fixing the parameters obtained from the previous fit (indicated by *).

\begin{tabular}{|c|c|c|c|c|c|c|}
\hline & Figure 8(a) & Figure $8(b)$ & Figures $8(\mathrm{c})-8(\mathrm{e})$ & Figure $8(f)$ & Figure $8(\mathrm{~g})$ & Figure $8(\mathrm{~h})-8(\mathrm{j})$ \\
\hline$\omega_{r} / 2 \pi(\mathrm{MHz})$ & $4461.8 \pm 0.5$ & $4476 \pm 3$ & $4476^{*}$ & $4447.8 \pm 0.1$ & $4463 \pm 1$ & $4463 *$ \\
\hline$\kappa_{\text {int }} / 2 \pi(\mathrm{MHz})$ & $\cdots$ & $\cdots$ & $8.4 \pm 0.2$ & $\cdots$ & $\cdots$ & $9.1 \pm 0.2$ \\
\hline$\kappa_{\mathrm{ext}} / 2 \pi(\mathrm{MHz})$ & $\cdots$ & $\cdots$ & $2.64 \pm 0.03$ & $\cdots$ & $\cdots$ & $2.64 \pm 0.03$ \\
\hline $2 t_{2} / 2 \pi(\mathrm{MHz})$ & $4447.7 \pm 0.5$ & $4451 \pm 2$ & $4451 *$ & . & $4463 \pm 2$ & $4463^{*}$ \\
\hline$g_{2} / 2 \pi(\mathrm{MHz})$ & $69.8 \pm 0.4$ & $69.0 \pm 0.5$ & $69.0 *$ & $\cdots$ & $69.3 \pm 0.5$ & $69.3 *$ \\
\hline$\gamma_{2,2}^{*} / 2 \pi(\mathrm{MHz})$ & $\cdots$ & $\cdots$ & $5.5 \pm 0.3$ & $\cdots$ & $\ldots$ & $4.0 \pm 0.6$ \\
\hline $2 t_{1} / 2 \pi(\mathrm{MHz})$ & $\ldots$ & $4452 \pm 1$ & $4452 *$ & $4450.1 \pm 0.2$ & $4461 \pm 1$ & $4461 *$ \\
\hline$g_{1} / 2 \pi(\mathrm{MHz})$ & $\cdots$ & $33.2 \pm 0.6$ & $33.2 *$ & $33.67 \pm 0.08$ & $34.7 \pm 0.4$ & $34.7 *$ \\
\hline$\gamma_{2,1}^{*} / 2 \pi(\mathrm{MHz})$ & $\cdots$ & $\cdots$ & $5.3 \pm 0.2$ & $\cdots$ & $\ldots$ & $6.9 \pm 0.3$ \\
\hline
\end{tabular}

master-equation simulations used for Figs. 2 and 7, but it needs far less computational effort. The main difference between the two methods is that the master-equation

TABLE IV. Extracted values from the fits performed on the data presented in Fig. 3(a) with taking the coupling rates obtained from the fits in Fig. 2. For Fig. 3(b), the coupling rates are extracted from the data in Fig. 8. In the caption of Fig. 3, we quote the Lamb-shifted qubit transitions as they are here in the table directly extracted from the Hamiltonian fit.

\begin{tabular}{lcc}
\hline \hline & Figure 3(a) & Figure 3(b) \\
\hline$\omega_{r} / 2 \pi(\mathrm{MHz})$ & $5432.9 \pm 0.9$ & $4650 \pm 3$ \\
$2 t_{1} / 2 \pi(\mathrm{MHz})$ & $5168.3 \pm 0.4$ & $4461 \pm 1$ \\
$2 t_{2} / 2 \pi(\mathrm{MHz})$ & $5164.1 \pm 0.3$ & $4440 \pm 1$ \\
\hline \hline
\end{tabular}

TABLE V. Parameters extracted from the fits performed in Fig. 9 with the coupling rate obtained from fits of the data in Fig. 2.

\begin{tabular}{lccc}
\hline \hline & Figure 9(a) & Figure 9(b) & Figure 9(c) \\
\hline$\omega_{r} / 2 \pi(\mathrm{MHz})$ & $4713 \pm 2$ & $4697 \pm 1$ & $4670 \pm 2$ \\
$2 t_{1} / 2 \pi(\mathrm{MHz})$ & $4392.2 \pm 0.3$ & $\ldots$ & $4493.1 \pm 0.3$ \\
$2 t_{2} / 2 \pi(\mathrm{MHz})$ & $\cdots$ & $4468.7 \pm 0.4$ & $4456.3 \pm 0.3$ \\
\hline \hline
\end{tabular}

simulation is more sensitive to residual detuning effects due to nonzero $\delta_{k}$ parameters than the Hamiltonian fitting, since it can also take into account the amplitude correctly, leading to an overall better fit.

Finally, for the data presented in Fig. 3, we extract frequencies of resonances in the experimental data using a simple Lorentzian fit. We then use the coupling parameters $g_{1,2}$ obtained from spectroscopy of the system configuration, shown in Fig. 8, and fit these data to a Hamiltonian model, Eq. (C5), where the only free parameters are the resonator and qubit frequencies as well as the scale of the detuning axis. Results are shown in Table IV. The same fitting procedure is used for the extracted parameters presented in Tables $\mathrm{V}$ and VI.

For the fits to the virtual photon-mediated coupling between the two DQDs in the dispersive regime (Fig. 4), we again start by extracting resonance frequencies from the data for both the DQD-like resonances as well as the detuned resonator. Using the DQD parameters extracted from the data in Fig. 3 as input, we then fit each of these data points to a Hamiltonian model to extract the DQD tunnel rates $2 t_{k}$ (assuming $\delta_{k}=0$ ) and resonator frequency $\omega_{r}$. Finally, we fit the $2 J$ data to a linear dependence in $1 / \Delta_{r}$, shown in Fig. 4(a). We observe linear dependence with a slight departure from the data.

TABLE VI. Extracted values from the Hamiltonian [Figs. 10(a), 10(b), 10(e), and 10(f)] and master-equation fits [Figs. 10(c), 10(d), $10(\mathrm{~g})$, and $10(\mathrm{~h})]$. Parameters that remain fixed are indicated by $*$.

\begin{tabular}{|c|c|c|c|c|c|c|}
\hline & Figure 10(a) & Figure 10(b) & $\begin{array}{l}\text { Figures } 10(\mathrm{c}) \\
\text { and } 10(\mathrm{~d})\end{array}$ & Figure $10(\mathrm{e})$ & Figure 10(f) & $\begin{array}{c}\text { Figures } 10(\mathrm{~g}) \\
\text { and } 10(\mathrm{~h})\end{array}$ \\
\hline$\omega_{r} / 2 \pi(\mathrm{MHz})$ & $5443.7 \pm 0.7$ & $5432.9 \pm 0.9$ & $5432.9 *$ & $5443 \pm 2$ & $5432 \pm 1$ & $5432 *$ \\
\hline $2 t_{1} / 2 \pi(\mathrm{MHz})$ & $5145.8 \pm 0.2$ & $5168.3 \pm 0.4$ & $5168.6 \pm 0.4$ & $5168.8 \pm 0.7$ & $5162.2 \pm 0.3$ & $5160.0 \pm 0.4$ \\
\hline$\gamma_{2,1} / 2 \pi(\mathrm{MHz})$ & $\cdots$ & $\cdots$ & $12.0 \pm 0.5$ & $\cdots$ & $\cdots$ & $13.7 \pm 0.6$ \\
\hline $2 t_{2} / 2 \pi(\mathrm{MHz})$ & $\cdots$ & $5162.8 \pm 0.7$ & $5164.1 \pm 0.3$ & $\cdots$ & $5159.7 \pm 0.5$ & $5156.0 \pm 0.5$ \\
\hline$\gamma_{2,2} / 2 \pi(\mathrm{MHz})$ & $\ldots$ & $\ldots$ & $8.8 \pm 0.5$ & $\cdots$ & $\ldots$ & $9.6 \pm 0.7$ \\
\hline
\end{tabular}


[1] R. Hanson, L. P. Kouwenhoven, J. R. Petta, S. Tarucha, and L. M. K. Vandersypen, Spins in Few-Electron Quantum Dots, Rev. Mod. Phys. 79, 1217 (2007).

[2] D. D. Awschalom, L. C. Bassett, A. S. Dzurak, E. L. Hu, and J. R. Petta, Quantum Spintronics: Engineering and Manipulating Atom-like Spins in Semiconductors, Science 339, 1174 (2013).

[3] L. M. K. Vandersypen, H. Bluhm, J. S. Clarke, A. S. Dzurak, R. Ishihara, A. Morello, D. J. Reilly, L. R. Schreiber, and M. Veldhorst, Interfacing Spin Qubits in Quantum Dots and Donors-Hot, Dense, and Coherent, npj Quantum Inf. 3, 34 (2017).

[4] G. Shinkai, T. Hayashi, T. Ota, and T. Fujisawa, Correlated Coherent Oscillations in Coupled Semiconductor Charge Qubits, Phys. Rev. Lett. 103, 056802 (2009).

[5] H.-O. Li et al., Conditional Rotation of Two Strongly Coupled Semiconductor Charge Qubits, Nat. Commun. 6, 7681 (2015).

[6] D. R. Ward, D. Kim, D. E Savage, M. G Lagally, R. H. Foote, M. Friesen, S. N. Coppersmith, and M. A. Eriksson, State-Conditional Coherent Charge Qubit Oscillations in a Si/SiGe Quadruple Quantum Dot, npj Quantum Inf. 2, 16032 (2016).

[7] M. D. Shulman, O. E. Dial, S. P. Harvey, H. Bluhm, V. Umansky, and A. Yacoby, Demonstration of Entanglement of Electrostatically Coupled Singlet-Triplet Qubits, Science 336, 202 (2012).

[8] M. Veldhorst et al., A Two-Qubit Logic Gate in Silicon, Nature (London) 526, 410 (2015).

[9] T. F. Watson et al., A Programmable Two-Qubit Quantum Processor in Silicon, Nature (London) 555, 633 (2018).

[10] D. M. Zajac, A. J. Sigillito, M. Russ, F. Borjans, J. M. Taylor, G. Burkard, and J. R. Petta, Resonantly Driven CNOT Gate for Electron Spins, Science 359, 439 (2018).

[11] T. A. Baart, T. Fujita, C. Reichl, W. Wegscheider, and L. M. K. Vandersypen, Coherent Spin-Exchange via a Quantum Mediator, Nat. Nanotechnol. 12, 26 (2016).

[12] G. Nicolí, M. S. Ferguson, C. Rossler, A. Wolfertz, G. Blatter, T. Ihn, K. Ensslin, C. Reichl, W. Wegscheider, and O. Zilberberg, Cavity-Mediated Coherent Coupling between Distant Quantum Dots, Phys. Rev. Lett. 120, 236801 (2018).

[13] R. P. G. McNeil, M. Kataoka, C. J. B. Ford, C. H. W. Barnes, D. Anderson, G. A. C. Jones, I. Farrer, and D. A. Ritchie, On-Demand Single-Electron Transfer between Distant Quantum Dots, Nature (London) 477, 439 (2011).

[14] T. Fujita, T. A. Baart, C. Reichl, W. Wegscheider, and L. M. K. Vandersypen, Coherent Shuttle of Electron-Spin States, npj Quantum Inf. 3, 22 (2017).

[15] A. Mills et al., Shuttling a Single Charge across a OneDimensional Array of Silicon Quantum Dots, arXiv: 1809.03976.

[16] L. Childress, A. S. Sørensen, and M. D. Lukin, Mesoscopic Cavity Quantum Electrodynamics with Quantum Dots, Phys. Rev. A 69, 042302 (2004).

[17] G. Burkard and A. İmamoğlu, Ultra-Long-Distance Interaction between Spin Qubits, Phys. Rev. B 74, 041307 (2006).

[18] X. Mi, J. V. Cady, D. M. Zajac, P. W. Deelman, and J. R. Petta, Strong Coupling of a Single Electron in Silicon to a Microwave Photon, Science 355, 156 (2017).
[19] A. Stockklauser, P. Scarlino, J. V. Koski, S. Gasparinetti, C. K. Andersen, C. Reichl, W. Wegscheider, T. Ihn, K. Ensslin, and A. Wallraff, Strong Coupling Cavity QED with Gate-Defined Double Quantum Dots Enabled by a High Impedance Resonator, Phys. Rev. X 7, 011030 (2017).

[20] X. Mi, M. Benito, S. Putz, D. M. Zajac, J. M. Taylor, G. Burkard, and J. R. Petta, A Coherent Spin-Photon Interface in Silicon, Nature (London) 555, 599 (2018).

[21] N. Samkharadze, G. Zheng, N. Kalhor, D. Brousse, A. Sammak, U. C. Mendes, A. Blais, G. Scappucci, and L. M. K. Vandersypen, Strong Spin-Photon Coupling in Silicon, Science 359, 1123 (2018).

[22] A. Wallraff, D. I. Schuster, A. Blais, L. Frunzio, R.-S. Huang, J. Majer, S. Kumar, S. M. Girvin, and R. J. Schoelkopf, Strong Coupling of a Single Photon to a Superconducting Qubit Using Circuit Quantum Electrodynamics, Nature (London) 431, 162 (2004).

[23] M. R. Delbecq, L. E. Bruhat, J. J. Viennot, S. Datta, A. Cottet, and T. Kontos, Photon-Mediated Interaction between Distant Quantum Dot Circuits, Nat. Commun. 4, 1400 (2013).

[24] G.-W. Deng et al., Coupling Two Distant Double Quantum Dots with a Microwave Resonator, Nano Lett. 15, 6620 (2015).

[25] J. S. Otterbach et al., Unsupervised Machine Learning on a Hybrid Quantum Computer, arXiv:1712.05771.

[26] J. Majer et al., Coupling Superconducting Qubits via a Cavity Bus, Nature (London) 449, 443 (2007).

[27] P. Campagne-Ibarcq et al., Deterministic Remote Entanglement of Superconducting Circuits through Microwave TwoPhoton Transitions, Phys. Rev. Lett. 120, 200501 (2018).

[28] P. Kurpiers et al., Deterministic Quantum State Transfer and Remote Entanglement Using Microwave Photons, Nature (London) 558, 264 (2018).

[29] C. Axline et al., On-Demand Quantum State Transfer and Entanglement between Remote Microwave Cavity Memories, Nat. Phys. 14, 705 (2018).

[30] P. Scarlino et al., All-Microwave Control and Dispersive Readout of Gate-Defined Quantum Dot Qubits in Circuit Quantum Electrodynamics, arXiv:1711.01906.

[31] X. Mi, J. V. Cady, D. M. Zajac, J. Stehlik, L. F. Edge, and J. R. Petta, Circuit Quantum Electrodynamics Architecture for Gate-Defined Quantum Dots in Silicon, Appl. Phys. Lett. 110, 043502 (2017).

[32] A. Blais, R. S. Huang, A. Wallraff, S. M. Girvin, and R. J. Schoelkopf, Cavity Quantum Electrodynamics for Superconducting Electrical Circuits: An Architecture for Quantum Computation, Phys. Rev. A 69, 062320 (2004).

[33] J. M. Fink, R. Bianchetti, M. Baur, M. Goppl, L. Steffen, S. Filipp, P. J. Leek, A. Blais, and A. Wallraff, Dressed Collective Qubit States and the Tavis-Cummings Model in Circuit QED, Phys. Rev. Lett. 103, 083601 (2009).

[34] A. J. Landig et al., Coherent Spin-Photon Coupling Using a Resonant Exchange Qubit, Nature (London) 560, 179 (2018).

[35] M. A. Sillanpää, J. I. Park, and R. W. Simmonds, Coherent Quantum State Storage and Transfer between Two Phase Qubits via a Resonant Cavity, Nature (London) 449, 438 (2007).

[36] L. DiCarlo et al., Demonstration of Two-Qubit Algorithms with a Superconducting Quantum Processor, Nature (London) 460, 240 (2009). 
[37] https://doi.org/10.3929/ethz-b-000288628.

[38] G. J. Dolan, Offset Masks for Lift-Off Photoprocessing, Appl. Phys. Lett. 31, 337 (1977).

[39] A. Stockklauser, Strong Coupling Circuit QED with Semiconductor Quantum Dots, Ph. D. thesis, ETH Zurich, 2017.

[40] J. Combes, J. Kerckhoff, and M. Sarovar, The SLH Framework for Modeling Quantum Input-Output Networks, Adv. Phys.: X 2, 784 (2017).

[41] C. Müller, J. Combes, A. R. Hamann, A. Fedorov, and T. M. Stace, Nonreciprocal Atomic Scattering: A Saturable,
Quantum Yagi-Uda Antenna, Phys. Rev. A 96, 053817 (2017).

[42] C. Müller and T. M. Stace, Deriving Lindblad Master Equations with Keldysh Diagrams: Correlated Gain and Loss in Higher Order Perturbation Theory, Phys. Rev. A 95, 013847 (2017).

[43] J. Lisenfeld, G. J. Grabovskij, C. Müller, J. H. Cole, G. Weiss, and A. V. Ustinov, Observation of Directly Interacting Coherent Two-Level Systems in an Amorphous Material, Nat. Commun. 6, 6182 (2015). 